\title{
Chapter 4 \\ Quantum Size Effects in the Growth and Properties of Ultrathin Metal Films, Alloys, and Related Low-Dimensional Structures
}

\author{
Y. Jia, M.M. Özer, H.H. Weitering, and Z. Zhang
}

\begin{abstract}
This chapter addresses the quantum mechanical nature of the formation, stability, and properties of ultrathin metal films, metallic alloys, and related lowdimensional structures, with $\mathrm{Pb}$ as a primary elemental example. The emphasis is on the contribution to the overall energetics from the electronic degrees of freedom of the low-dimensional systems. As a metal film reduces its thickness, the competition between quantum confinement, charge spilling, and Friedel oscillations, all of electronic origin, can dictate whether an atomically smooth film is marginally, critically, or magically stable or unstable against roughening during the growth of such metal films. The "electronic growth" mode as emphasized here serves as an intriguing addition to the three well-established classic modes of crystal growth. In exploring electronic growth, $\mathrm{Pb}(111)$ films represent a particularly compelling example, not only because their stability exhibits unusually strong quantum oscillations but also because their physical and chemical properties can be tuned with great precision by controlling the film thickness or the chemical composition. Recent advances and the perpectives in this active area of film growth will be reviewed, with results from both theoretical and experimental studies.
\end{abstract}

\subsection{Historical Review}

Low-dimensional materials have been an important platform for the discovery of novel quantum phenomena, such as the quantized conductance and quantum Hall effects in semiconductor heterostructures [1] and giant magneto-resistance in metallic magnetic superlattices [2]. In these examples, fabrication of the low-dimensional medium was accomplished via classical thin film growth; yet the emerging properties are clearly quantum mechanical in nature. More recently, it has been shown that the size quantization of itinerant electrons in an ultrathin metal film also plays a decisive role in the early formation stages and kinetic stability of the films [3-9].

\footnotetext{
Y. Jia $(\bowtie)$

School of Physics and Engineering, Zhengzhou University, Zhengzhou, Henan 450052, China; Materials Science and Technology Division, Oak Ridge National Laboratory, Oak Ridge, TN 37831, USA

e-mail: jiayu@zzu.edu.cn
} 
The ability to exploit quantum mechanical principles while tuning and assembling nanostructures offers a whole new range of opportunities for exploring the physical and chemical properties of functional nanomaterials with precisely controlled dimensions [10-15].

Atomically flat metal thin films on a proper substrate, such as $\mathrm{Ag} / \mathrm{GaAs}(110)$, $\mathrm{Pb} / \mathrm{Si}(111), \mathrm{Pb} / \mathrm{Ge}(111), \mathrm{Al}(110) / \mathrm{Si}(111), \mathrm{In} / \mathrm{Si}(111)$, and $\mathrm{Mg}(0001) / \mathrm{Si}(111)$ films, have been the model systems for exploring electronically driven self-assembly, a process dubbed "electronic growth" or "quantum growth" [4]. In these systems, the interplay between the quantum size effect and the morphological evolution of the films received considerable attention following the discovery of "magic thicknesses" or "critical thicknesses" and perferred island heights in ultrathin metal films grown at moderately low tempertures. In particular, $\mathrm{Pb}$ is a soft metal and hence electronic effects in the formation stages of the film could easily overwhelm lattice strain contributions to the total free energy of the film [16]. An added consideration is that the Fermi wavelength $\lambda_{\mathrm{F}}$ is nearly commensurate with the interatomic layer spacing $d$ along the $\langle 111\rangle$ direction, which amplifies the role of quantum size effects and Friedel oscillations on the growth mode of the $\mathrm{Pb}$ films.

Studies of the quantum size effect (QSE) in thin films date back to the 1970s. Jaklevic and Lambe first observed the QSE by means of tunneling experiments in metal-oxide-metal junctions [17]. Schulte carried out self-consistent studies of thin films and showed that the work function oscillates with the thickness of the films [18]. Such oscillations were also confirmed beyond the jellium model by Feibelman [19], who also found oscillations in the surface energy.

In the 1990s, researchers found that atomically smooth metal films on semiconductors are sometimes formed at low temperatures, under growth conditions that seem to be far away from thermodynamic equilibrium [3]. However, because the adatom diffusion still appears to be significant in this growth regime, thermodynamic considerations can still be applied at least locally. This novel growth mode is qualitatively different from the three well-established classical growth modes [20] and appears to be related to quantum size effects that provide a non-trivial thicknessdependent contribution to the free energy of the film, one that easily overwhelms the strain contributions due to lattice mismatch. This has led to the surprising observation that smooth film growth in the quantum regime is possible only above a certain critical thickness $[8,21,22]$. Qualitatively, this newly defined critical thickness is just the inverse of the transition at the critical thickness defined in the classic Stranski-Krastanov (SK) growth mode [23].

The first striking example of the quantum growth mode of $\mathrm{Ag}$ on GaAs was revealed in a two-step process [3], deposition at low temperature and subsequent annealing to room temperature. At very low temperature, since the mobility is very low, only tiny grains or "nanoclusters" are formed as opposed to smooth films or large 3D clusters. Upon heating the sample to room temperature, the atoms acquired sufficient mobility to self-organize into an atomically smooth film with a thickness of six monolayers (ML). If the nominal deposition amount is slightly less, then the film contains large holes exposing the bare GaAs substrate. Evidently, the "underdosed" Ag film phase separates into a $6 \mathrm{ML}$ thick Ag film and holes exposing the 
bare substrate. This indicates that the atomically flat $6 \mathrm{ML}$ thick Ag film constitutes a particularly stable morphology.

Similar behavior has been observed in both $\mathrm{Ag}$ and $\mathrm{Pb}$ films grown on $\mathrm{Si}(111)$ at low temperature [7, 8]. Scanning tunneling microscopy (STM) and spot profile analysis low energy electron diffraction measurements of $\mathrm{Pb} / \mathrm{Si}(111)$ revealed the presence of large islands with flat-tops, steep edges, and strongly preferred heights $[24,25]$. Budde et al. have observed that $\mathrm{Pb}$ islands of seven monolayers are preferred to form on the $\mathrm{Si}(111)-(7 \times 7)$ substrate at low temperatures with low energy electron diffraction (LEED) [21]. Su et al. [24] confirmed this observation with STM. The existence of competing 4 or 5, 7 and $9 \mathrm{ML}$ island heights was summarized in a "kinetic phase diagram" for nominal coverages up to $10 \mathrm{ML}$ and temperatures between 120 and $250 \mathrm{~K}$ [25]. Hong et al. [22] studied the same system using realtime in situ X-ray diffraction, and their results show that the growth switches to a layer-by-layer mode for films of over five monolayers. Özer et al. performed a comprehensive STM study of Pb films on three different semiconducting substrates, $\mathrm{Si}(111)-7 \times 7, \operatorname{Si}(111)-\sqrt{3} \times \sqrt{3} R 30^{\circ}-\alpha$, and $\mathrm{Ge}(111)-\sqrt{3} \times \sqrt{3}-\alpha$. It is found that $\mathrm{Pb}$ films have the same re-entrant bilayer-by-bilayer growth mode on these three different substrates.

Remarkably, the quantum stability of the meal films, derived from single or nearly free electron pictures, also influences a wide variety of physical and chemical properties. In this chapter, we will first discuss the role of the quantum size effect in the stability of thin metallic films from both the free electron model and the density functional theory perspectives, and we will highlight some of the key experimental observations for mostly $\mathrm{Pb}$ and $\mathrm{Mg}$. The second part of this review will be devoted to the structural, physical, and chemical properties of the films, specifically lattice relaxations and work functions, superconductivity, and surface chemical reactivity. We refer to the chapter by Tringides and Altman in this volume [pp. XX-XX] with regard to QSE on growth kinetics, particularly the various atomistic rates on different metal films and flat-top nanoislands. We also refer to a somewhat related recent review from our collaborative team [26], which emphasized more on the experimental aspects of the subjects.

\subsection{The Quantum Size Effect}

\subsubsection{Quantum Size Effect and Electronic Growth}

When a metal film becomes very thin, there will be three central ingredients that can influence the stability of the film on the supporting substrate, namely quantum comfinement, charge spilling, and interface-induced Friedel oscillations [4]. Electronic confinement within the metal overlayer can mediate an effective repulsive force between the interface and the surface. Electron transfer from the overlayer to the substrate leads to an attactive force between the two interfaces, acting to distabilize the overlayer [16]. Interface or surface-induced Friedel oscillations in 
electron density can further impose an oscillatory modulation onto the two previous interactions.

Generally, for most metals, the Fermi wavelength is comparable to the lattice spacing in a given direction. This means that if a metal film is only several atomic layers thick, the motion of the nearly free electrons perpendicular to the film will resemble that of the famous "particle-in-a-box" in introductory quantum mechanics. When the "free" electrons are squeezed into a box with hard wall barriers (referring to one dimension for simplicity), the momentum wave vector should become quantized such that an integer multiple of half-wavelengths can fit inside the box. In a very thin film the wave function of an itinerant electron is expected to obey the same quantization condition along the film normal, the total phase difference accumulated by the reflections must be a number of $2 \pi$ according to the Sommerfeld-Bohr quantization rule:

$$
2 k_{\mathrm{z}} d+\Phi_{1}+\Phi_{2}=2 \pi n
$$

here $k_{\mathrm{z}}$ is the allowed wave vector component normal to the surface, $n$ takes on integer values, $d$ is the thickness of the films, and $\Phi_{1}$ and $\Phi_{2}$ are the phase terms that account for wavefunction leakage beyond the potential steps at the film boundaries, for the vacuum films on both sides, the $\Phi_{1}$ and $\Phi_{2}$ are taken to be $\pi$. Furthermore we have $k_{\mathrm{z}}=n \pi / d$ or, equivalently, $d=n \lambda / 2$, where $n$ is a non-zero, positive integer. The parallel momentum states, however, are still represented by two-dimensional (2D) Bloch waves with a free electron-like dispersion, $E\left(\vec{k}_{0}\right)=\hbar^{2} k_{0}^{2} / 2 m^{*}$, where $m^{*}$ is the effective mass. In turn, the discretization of the electronic energy band can lead to an oscillatory dependence of the film's total energy on its thickness, instead of the linear dependence on thickness for very thick films. This oscillatory behavior implies that a thin film of certain layers may be energetically favored over other layers, opening a window of opportunity that an atomically flat film will form upon annealing [4, 16, 27] (see Figs. 4.1 and 4.2).

A more accurate analysis of quantum size effect physics requires first-principles electronic structure calculations as a function of the film thickness. Such first-

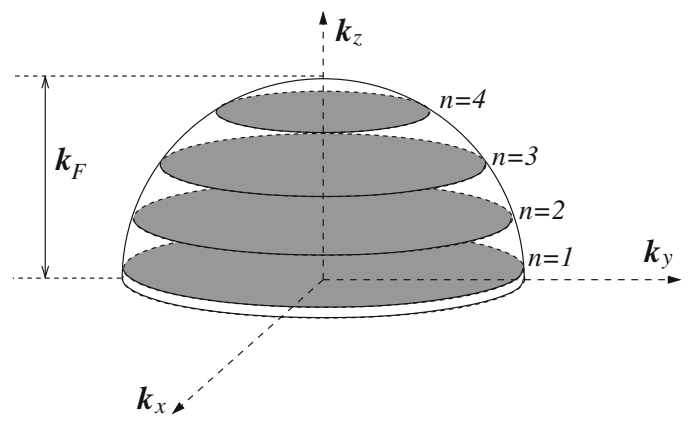

Fig. 4.1 Energy subbands of a metallic thin film 

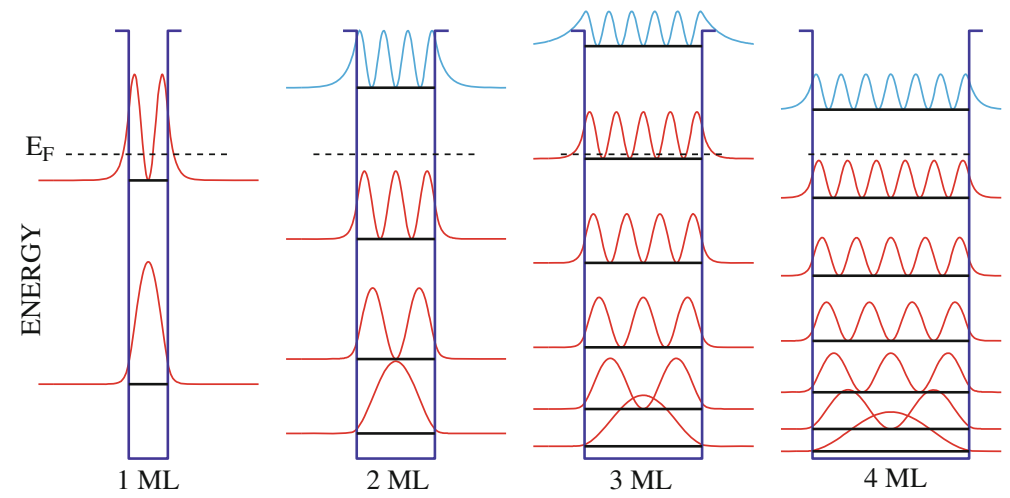

THICKNESS

Fig. 4.2 Sketch of confinement of electrons in ultrathin metallic films

principles calculations confirm the qualitative predictions from the nearly free electron model. The thickness-dependent surface energy and work function also follow from such calculations and exhibit damped oscillations as a function of the film thickness, with periods depending on the specific systems.

\subsubsection{Surface Energy and the Stability of Films}

We first discuss the criterion of stablity, that is, how to use the total energies to determine the stability of a metallic film or the possible outcome of an annealing experiment. As a given amount of a certain material is deposited on a substrate, many different morphologies may result. The case of continuous film is illustrated in Fig. 4.3. During annealing at certain temperatures, the film will likely change its morphology. The system always tends to seek a morphology of lower energy when it is allowed by growth kinetics. The configuration that is a local minimum in the system energy is relatively stable and will likely be the outcome of annealing.

The total energy $E_{\mathrm{t}}(L)$ of a thin metallic film can be obtained, within the free electron model, by summing over the single particle energies $E_{n}\left(k_{0}, L\right)$ below the

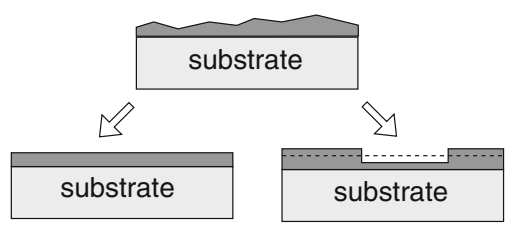

Fig. 4.3 Morphological evolution of continuous thin films. The top is a rough film before annealing; after annealing, the film may become atomically flat (bottom left) or develop into a film with two different heights (bottom right). In these cases, the contact area between the film and the substrate is conserved during the morphological evolution [27] 
Fermi level $E_{\mathrm{t}}(L)$ of the film [27]. Here, $n$ represents the subband index and $L$ is the film thickness in monolayers ( $d=L d_{0}, d_{0}$ being the interlayer spacing). This total energy determines the thermodynamic stability of the film at low temperatures. One usually subtracts the bulk energy to obtain the surface energy $E_{\mathrm{S}}(L)$ per unit area. A thin film of $L$ monolayers is (meta) stable if the electronic compressibility $\partial^{2} E_{\mathrm{S}} / \partial L^{2}>0$. Under this condition, small-scale roughness in the film will be suppressed, provided that the atom mobility is high enough. A film is unstable if $\partial^{2} E_{\mathrm{s}} / \partial L^{2}<0$, in which case the system can achieve a lower energy state by developing a mixed phase of different film thicknesses. A critical thickness $L_{\mathrm{c}}$ can be defined if the film is stable for $L \geq L_{\mathrm{c}}$ and unstable for $L<L_{\mathrm{c}}$ or the other way around. A magic thickness $L_{\mathrm{m}}$ can be defined if $E_{\mathrm{s}}(L)$ has a downward cusp at $L_{\mathrm{m}}$, meaning that the film is unstable on both sides of $L_{\mathrm{m}}$ [4].

For discrete layer thicknesses, this criterion is sometimes recast as a criterion for the discrete "second difference" of $E_{\mathrm{S}}(L)$ :

$$
\mathrm{d}^{2} E_{\mathrm{S}}(L)=E_{\mathrm{S}}(L+1)+E_{\mathrm{S}}(L-1)-2 E_{\mathrm{S}}(L)
$$

which should also be positive for a stable film of thickness $L \mathrm{~d}_{0}$. This criterion should be viewed as a criterion for the local stability of the film. It does not rule out phase separation into film regions with a thickness of $L \pm 2$. Global stability of a closed film morphology requires that $E_{\mathrm{S}}(L)$ is convex (or $\partial^{2} E_{\mathrm{S}} / \partial L^{2}>0$ ) over a wide coverage range. This is indeed the case for the nearly free electron model if one ignores the small quantum oscillations arising from the band discretization. These small quantum oscillations could make the function $E_{\mathrm{S}}(L)$ locally convex, which in turn should lead to phase separation into preferred thicknesses, a phenomenon that is sometimes referred to as "quantum phase separation" [22].

\subsection{Understanding the QSE Within Nearly Free Electron Models}

\subsubsection{Nearly Free Electron Model}

To illustrate the basic physics of how quantum size effects can affect the growth and stability of a metal film, we focus on the total electronic energy as a function of layer thickness starting with non-interacting electrons that are vertically confined by a square well potential, this is the so-called nearly free electron model. In this model, a metal film can be described with a free electron gas confined to an infinte quantum well. In general, the total energy $E_{\mathrm{t}}$ of a freestanding metallic thin film with thickness $d$ determines the thermodynamic stablity. It can be shown that [27]

$$
E_{\mathrm{t}}=\frac{m_{e} S}{2 \pi \hbar^{2}} \sum_{n \leq N}\left(E_{\mathrm{F}}^{2}-E_{n}^{2}\right)
$$

And the surface energy $E_{\mathrm{S}}$ is 


$$
E_{\mathrm{S}}(d)=\frac{k_{\mathrm{F}}^{2} E_{\mathrm{F}}}{4 \pi}\left\{\sum_{n \leq N} \frac{E_{\mathrm{F}}^{2}-E_{n}^{2}}{E_{\mathrm{F}}^{2}}-\frac{4}{5} \frac{\mathrm{d} k_{\mathrm{F}}}{\pi}\right\}
$$

where $E_{\mathrm{F}}, E_{\mathrm{f}}$, and $k_{\mathrm{F}}$ are the Fermi level, Fermi energy, and Fermi wave number, respectively. $S$ is the surface area of the films.

According to (4.3) and (4.4), one noticeable result for such a nearly free electron model is that the Fermi energy of a free electron slab is pushed to higher energy as the film thickness decreases, which is due to the increase of the kinetic energy (Fig. 4.4a and b) [4, 27-29]. In addition, the film's Fermi energy oscillates as a function of thickness, as marked by a series of cusps that are separated by a thickness interval of $\lambda_{\mathrm{F}} / 2$. This is related to the fact that the level spacing decreases with increasing thickness. As the film thickness increases, the Fermi energy exhibits a cusp when an empty subband begins to shift through the Fermi level. At the Fermi

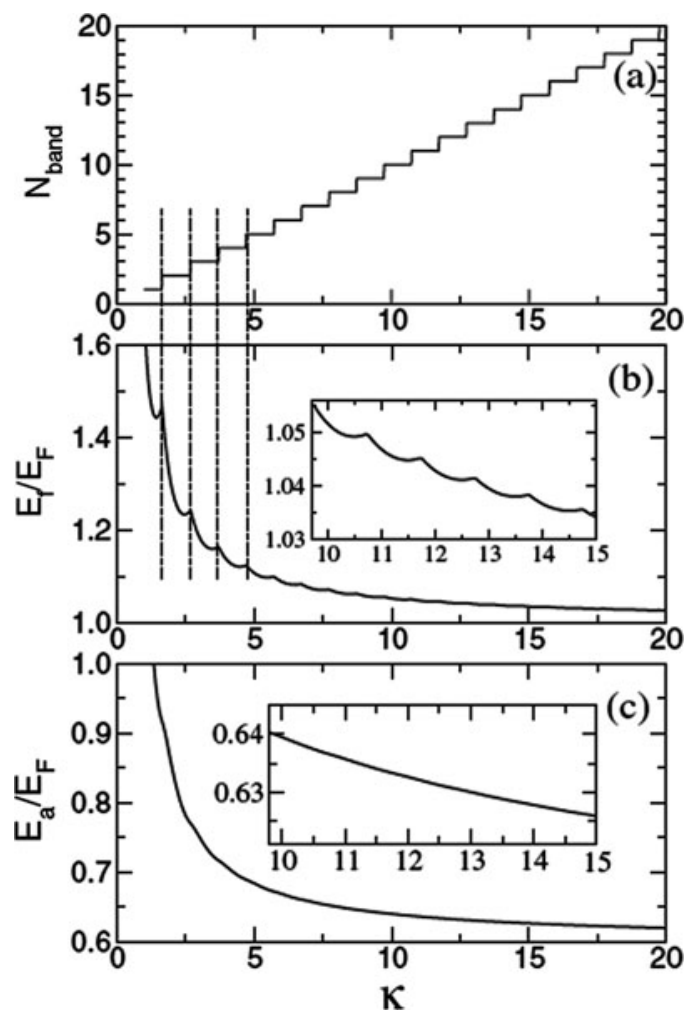

Fig. 4.4 (a) Number of subbands below the Fermi energy. (b) Fermi energy $E_{\mathrm{f}}$ and (c) surface energy per electron $E a$ as a function of a metallic thin film. The interfaces are modeled as infinite energy barriers, showing the increase of the Fermi energy at low film thicknesses as well as the superimposed oscillations with a period of $\lambda_{\mathrm{F}} / 2$. The film thickness is labeled by the dimensionless number $\kappa=\mathrm{d} k_{\mathrm{F}} / \pi$. Reproduced from [27]. Insets show the enlarged portions of $E_{\mathrm{f}}$ and $E_{a}$. The dashed lines indicate the correspondence between the emergence of a new subband and a cusp 
level, the general quantization condition $d=n \lambda / 2$ becomes $d=n \lambda_{\mathrm{F}} / 2$ and, consequently, the cusps in $E_{\mathrm{F}}(d)$ are separated by a thickness $\lambda_{\mathrm{F}} / 2$. Because of the $\lambda_{\mathrm{F}} / 2$ oscillations of the Fermi level, the cohesive energy of the slab oscillates as well.

To be specific, the five different metallic films which represent three different types of stability, with their second differences are shown in Fig. 4.5, revealing that

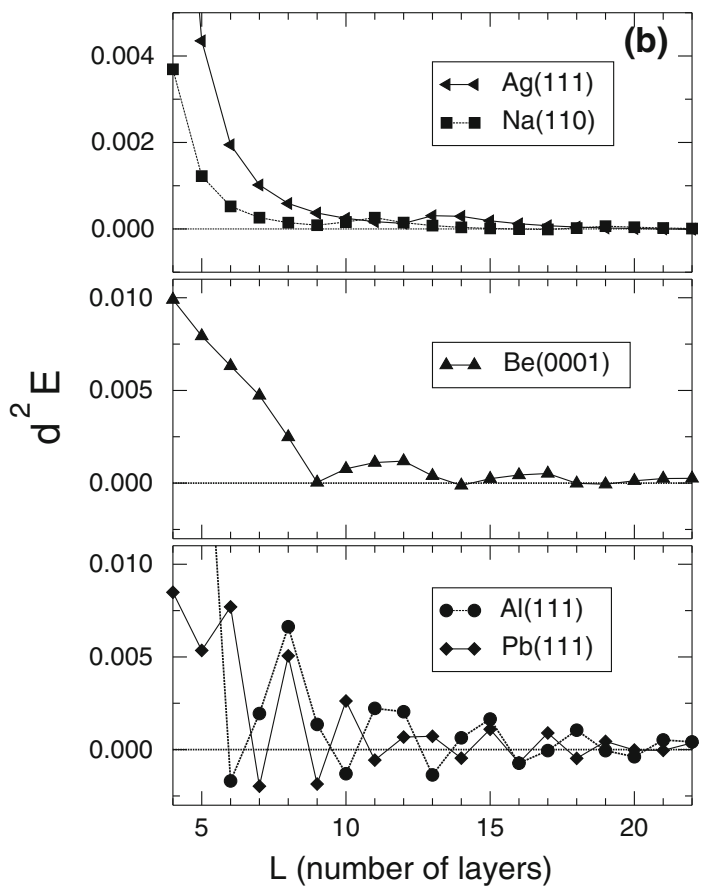

(a)

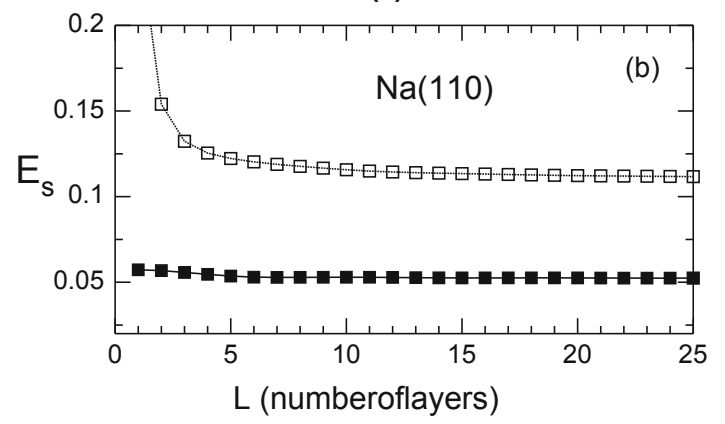

(b)

Fig. 4.5 Surface energy and discrete second differences: (a) The infinite potential well captures the qualitative differences in the local stability of $\mathrm{Ag}(111), \mathrm{Na}(110), \mathrm{Be}(0001), \mathrm{Al}(111)$, and $\mathrm{Pb}(111)$ films. (b) Freestanding $\mathrm{Na}(110)$ films exhibit a dramatic reduction of the kinetic energy when using finite energy barriers (black squares) as opposed to infinite barriers (open squares). The kinetic energy lowering is due to charge spilling and is especially profound at small thicknesses. Reproduced from [27] 
the films are very different from each other. The alkali metal $\mathrm{Na}(110)$ and transition metal $\mathrm{Ag}(111)$ films are the first type of stability, their surface energy become bulklike very quickly: beyond five monolayers $(L>5)$, their $\mathrm{d}^{2} E_{\mathrm{s}}$ is already very small. Another type is alkaline earth metal films, such as $\mathrm{Mg}(0001)$ and $\mathrm{Be}(0001)$ films; the surface energy of $\mathrm{Be}(0001)$ films decays into bulk slower; despite the apparent oscillations, $\mathrm{Be}(0001)$ films of different monolayers are stable since its second difference $\mathrm{d}^{2} E_{\mathrm{s}}$ is almost always positive. Even its second difference $\mathrm{d}^{2} E_{\mathrm{s}}$ becomes negative at a few thicknesses, its absolute value is very small and does not indicate a strong instability. This implies that, according to the computation, it is very hard to grow atomically flat $\mathrm{Be}(0001)$ of any thickness in experiments. However, $\mathrm{Al}(111)$ and $\mathrm{Pb}(111)$ films are very different from the above two types of films. First, their second difference $\mathrm{d}^{2} E_{\mathrm{s}}$ decays even slower. Second, their $\mathrm{d}^{2} E_{\mathrm{s}}$ oscillates around zero with $\mathrm{d}^{2} E_{\mathrm{s}}$ possibly being negative. For example, $\mathrm{Al}(111)$ films have negative $\mathrm{d}^{2} E_{\mathrm{s}}$ at $L=5,10,13$, and 16 monolayers, which means that $\mathrm{Al}(111)$ films are unstable at these thickness. In other words, unlike $\mathrm{Be}(0001)$, the oscillations here in $\mathrm{d}^{2} E_{\mathrm{s}}$ imply oscillations in the film stability for $\mathrm{Al}(111)$ and $\mathrm{Pb}(111)$ films. Besides the apparent similarity, $\mathrm{Al}(111)$ and $\mathrm{Pb}(111)$ films have different oscillation patterns in the film stability. The stability of $\mathrm{Pb}(111)$ films oscillates in an even-odd fashion interrupted by crossovers. This is exactly the oscillation pattern observed in the stability of $\mathrm{Pb}(111)$ film in [15], although there exists difference in which layers are stable due to the substrate effects in the experimental studies. Considering how crude the present model is, the agreement is quite amazing. Moreover, the amplitude of the oscillations in $\mathrm{d}^{2} E_{\mathrm{s}}$ matches well with the ab initio calculations in. [10, 15, 30, 33] and, more importantly, in thin film growth experiments [8, 31]. The oscillation pattern in film stability is determined by the ratio between the Fermi wavelength $\lambda_{\mathrm{F}}$ and the layer spacing $d_{0}$ as we will discuss in the following sections. For $\mathrm{Pb}(111)$ films, we have $\lambda_{\mathrm{F}} / 2: d_{0}=1: 1.44 \approx 2: 3$; for $\mathrm{Al}(111)$ films, we have $\lambda_{\mathrm{F}} / 2: d_{0}=1: 1.3 \approx 3: 4$.

The model can be improved by changing the infinite potential barriers with finite barriers, taking into account the lattice potential, allowing the electron wave function to spill into the vacuum and substrate regions [27, 30]. Figure 4.6 presents the Fermi energy of a $\mathrm{Pb}(111)$ film as a function of thickness with different boundaries. In the infinite well model, when the thickness of the films is exactly equal to $N d_{0}$, $E_{\mathrm{F}}(d)$ rises drastically as the film thickness decreases to compensate for the areas of electron depletion near the film boundaries. However, with a finite well, as the energy barrier decreases, the less confinement felt by the free electrons and the electrons can spill out of the film more easily, so such compensation is unnecessary. Therefore, the Fermi energies $E_{\mathrm{f}}$ of the film decrease accordingly to its bulk value. At the same time, the slopes of the energies decreasing with thickness become less steep as finite well decreases. This makes the oscillations in Fermi energy $E_{\mathrm{f}}$ to appear more pronounced. On the other hand, in the infinite well but allowing the electron density to allow to spill out the classical film boundaries, the result is very similar to the case of finite well. In addition, as indicated by the dashed line in Fig. 4.6, the barrier height of the well shifts the cusp positions, which is known as phase shift [32]. 


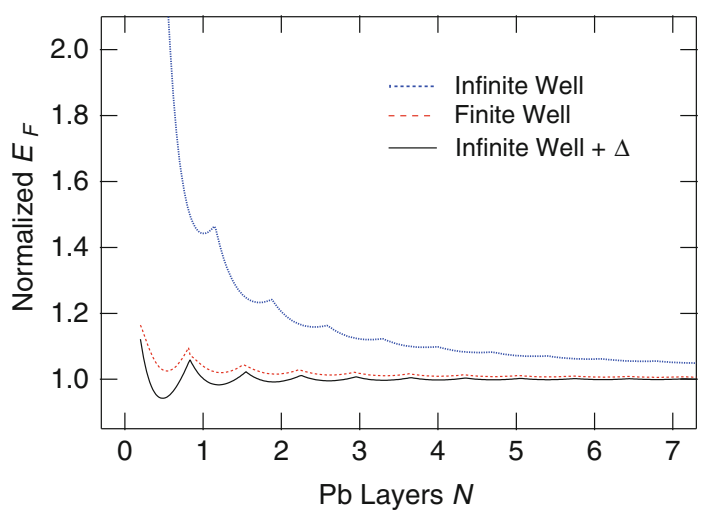

Fig. 4.6 The Fermi energy of $\mathrm{Pb}(111)$ films as a function of the thickness, normalized to its bulk free electron value. Reproduced from [29]

\subsubsection{Beyond the Nearly Free Electron Model}

The next step is to include exchange and correlation effects within the density functional theory (DFT) formalism. From Schulte's calculations [18] it is evident that the main feature, such as the $\lambda_{\mathrm{F}} / 2$ oscillation of the Fermi level remains preserved in DFT. However, in these calculations the oscillation of the Fermi energy is caused primarily by a corresponding variation in the effective potential, whereas the variation in the kinetic energy with thickness is very small. This has implications for the interpretation of the calculated work function oscillations. The latter are caused primarily by the oscillations in the surface dipole as a function of the layer thickness, not by periodic variations of the Fermi energy.

The effective potential in DFT is computed self-consistently and contains the electrostatic potential and the local exchange-correlation potential. Interestingly, the electrostatic potential in the jellium calculations reveals significant oscillations or Friedel oscillations in the electron density, due to the presence of two surfaces. The oscillations in the electrostatic potential are partially offset by corresponding oscillations in the exchange correlation potential so that the oscillations in the effective potential are weaker than those of the electrostatic potential. As we will show, however, Friedel oscillations can still play a decisive role in the thin film stability.

It should be noted that the properties (e.g., work function, potentials, total energy) of a jellium slab are derived from universal curves that only scale with the electron density or value of $r_{\mathrm{s}}$. To assess the predictive capability for real materials, the universal curves must be sampled with the discrete atomic-layer spacing of each material. It is not a priori obvious whether the $\lambda_{\mathrm{F}} / 2$ quantum oscillations can be observed experimentally. For instance, if the atomic layer spacing $d_{0}$ was perfectly commensurate with $\lambda_{\mathrm{F}} / 2$, then there would be no oscillations at all.

Nowadays it is possible to go beyond jellium calculations and extract the properties from advanced DFT calculations that include the fully relaxed atomic coordinates $[6,9]$. The predictive capabilities of these calculations are amazingly accurate. 
However, this benefit of accuracy does not necessarily imply a higher level of transparency of the underlying physical principles. Simple model calculations remain invaluable in assessing the basic principles of thermodynamic stability in the thin film quantum regime.

Finally, it has been shown that the oscillations become more profound if one reduces the confinement by lowering the potential barriers at both interfaces $[4,5,27]$. As expected, the effects of charge spilling are most profound in the thinnest films, say less than $5 \mathrm{ML}$. This effect is likely to be very important for thin metal films on semiconductors where the confinement at the interface arises from the Schottky barrier height. The latter is usually much smaller than the potential barrier on the vacuum side (i.e., work function) and, hence, the stability criterion for a freestanding metal slab and that of a metal on a semiconductor could be qualitatively different. Indeed, whereas the quantum mechanically confined electron motion produces an energy contribution that helps to stabilize the film against roughening, charge spilling results in a contribution that potentially destabilizes the metal film. The latter contribution is roughly estimated from the charge transfer across the metal/semiconductor interface, which also increases with increased confinement. The balance between these two energy contributions defines the new critical thickness below which smooth film growth should be impossible [4, 27]. The argument can only be qualitative, however, because it lacks self-consistency.

\subsection{Quantum Size Effects in $\mathrm{Pb}(111)$ Films}

\subsubsection{Re-entrant Bilayer-by-Bilayer Growth Behaviors of Pb Films: Experimental Results}

When ultrathin $\mathrm{Pb}$ films grow on a semiconductor or metal substrate, such as $\mathrm{Si}(111), \mathrm{Ge}(111)$, and $\mathrm{Cu}(111)$, the quantum size effect prevails over the strain effect, despite the large lattice mismatch that always exists. Experimentally, the bilayer oscillations were first identified with helium atom scattering (HAS) [33] and had been correctly attributed to the bilayer-by-bilayer growth of $\mathrm{Pb}(111)$ although later an alternative explanation was offered by the same group in which the bilayer oscillations in HAS were attributed to bilayer oscillations in the charge spilling during layer-by-layer growth [34].

Comprehensive and comparative STM studies of $\mathrm{Pb}$ growth on three different types of $\mathrm{Ge}(111)$ and $\operatorname{Si}(111)$ substrates, namely $\operatorname{Si}(111) \sqrt{3} \times \sqrt{3}, \operatorname{Si}(111) 7 \times 7$, and $\operatorname{Ge}(111) \sqrt{3} \times \sqrt{3}$, respectively, have been carried out [8]. The results show that quantum growth can be exploited to produce atomically smooth $\mathrm{Pb}$ films over mesoscopic length scales on all three substrates. The minimum coverage for smooth film growth or critical thickness $t_{\mathrm{C}}=5 \mathrm{ML}$. In the smooth growth regime, there exists an intriguing re-entrant bilayer-by-bilayer (RBBB)_mode, characterized by strong preference of bilayer growth with periodic interruption of monolayer or trilayer growth. 
Figure 4.7a shows a $500 \times 500 \mathrm{~nm}$ STM image of a Pb film on $\mathrm{Si}(111)$ substrate. Flat-topped islands rising 4 or $5 \mathrm{ML}$ above the wetting layer can be seen; no other island heights have been observed. In this coverage range, $\mathrm{Pb}$ islands never merge to form a continuous film. Figure $4.7 \mathrm{~b}$ shows a $500 \times 500 \mathrm{~nm}$ image of an almost
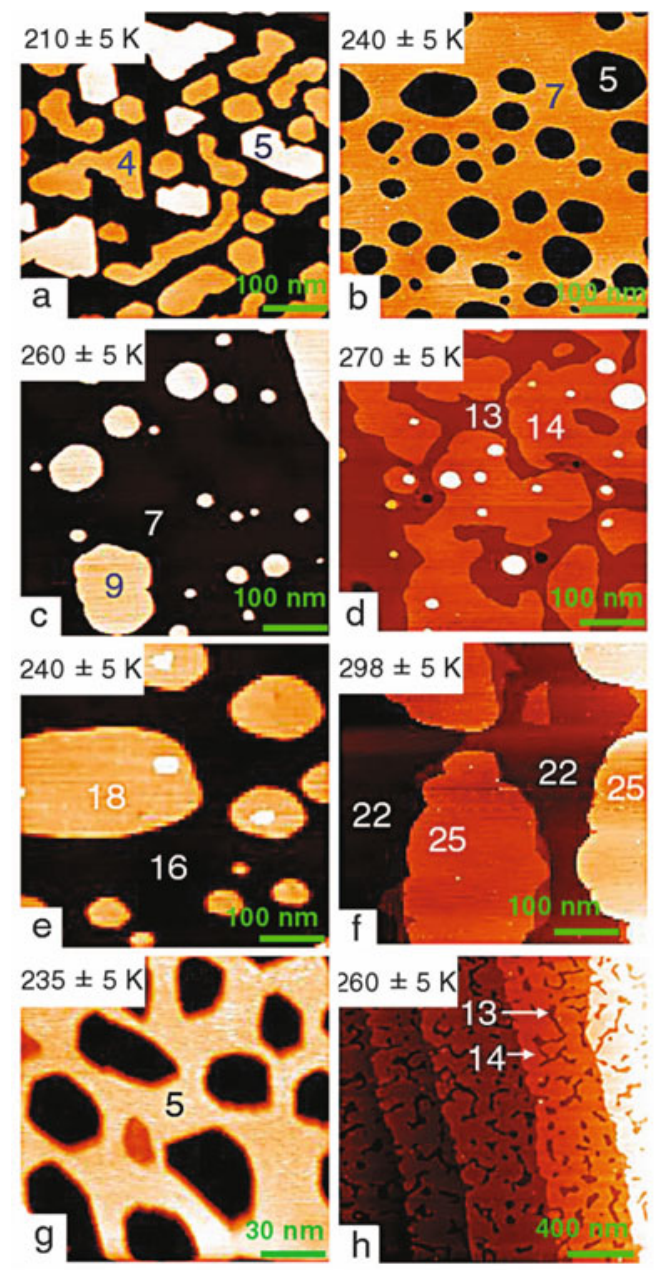

\begin{tabular}{|c|c|c|c|c|c|c|c|c|c|c|c|}
\hline Interface & \multicolumn{11}{|c|}{ Observed Layer Thickness } \\
\hline Si(111)7×7-Pb & 5 & 7 & 9 & 11 & 13 & 14 & 16 & 18 & 20 & & \\
\hline $\operatorname{Si}(111)(\sqrt{3} \times \sqrt{3})-\alpha$ & 5 & 7 & 9 & 11 & 13 & 14 & 16 & 18 & 20 & 22 & 25 \\
\hline $\mathrm{Ge}(111)(\sqrt{3} \times \sqrt{3})-\alpha$ & 5 & 7 & 9 & 11 & 13 & 14 & 16 & 18 & 20 & & \\
\hline
\end{tabular}

Fig. 4.7 $\mathrm{STM}$ images of $\mathrm{Pb}$ on (a-f) $\mathrm{Si}(111) \sqrt{3} \times \sqrt{3}-R 30^{\circ}-\mathrm{Pb},(\mathbf{g}) \mathrm{Ge}(111)-\sqrt{3} \times \sqrt{3}-R 30^{\circ}-$ $\mathrm{Pb}$, and (h) $\mathrm{Si}(111)-7 \times 7$. Layer thicknesses are indicated in each panel and are measured with respect to the wetting layer. The table summarizes the observed thicknesses for each interface with even-odd crossovers indicated in bold italics. Image sizes and postannealing temperatures are also indicated in $\mathbf{a}-\mathbf{h}$. From [8] 
continuous $7 \mathrm{ML} \mathrm{Pb}$ film with $2 \mathrm{ML}$ deep voids. It is clear that in this coverage regime, the $5 \mathrm{ML}$ film is atomically flat and continuous on a mesoscopic length scale while the larger part of the $5 \mathrm{ML}$ surface is covered with an additional bilayer of $\mathrm{Pb}$, making the total film thickness to be $7 \mathrm{ML}$. The 5 and $7 \mathrm{ML}$ terraces neither possess single monolayer steps nor single monolayer voids, thus confirming the onset of perfect bilayer growth on top of the closed $5 \mathrm{ML}$ film. Figure $4.7 \mathrm{c}$ reveals $2 \mathrm{ML}$ high islands on the $7 \mathrm{ML}$ films. Again, the absence of voids or monolayer high islands on the $7 \mathrm{ML}$ film shows that the growth proceeds in a perfect bilayer-by-bilayer mode. Bilayer growth continues until the coverage reaches $13 \mathrm{ML}$. Figure $4.1 \mathrm{~d}$ shows a 13 ML high film with monatomic layer high islands (14 ML) residing on top and tiny amounts of $16 \mathrm{ML}$ high islands. After this intermission, near-perfect bilayer growth resumes at $14 \mathrm{ML}$, ending at $22 \mathrm{ML}$. Figure $4.7 \mathrm{e}$ shows the bilayer height steps on a $16 \mathrm{ML}$ film. Figure $4.7 \mathrm{f}$ shows $3 \mathrm{ML}$ high steps on top of a $22 \mathrm{ML}$ high film, indicating a trilayer intermission. In all, $\mathrm{Pb}$ grows in a perfect bilayer-by-bilayer mode from 5 to $13 \mathrm{ML}$ and from 14 to $22 \mathrm{ML}$. The stable thicknesses are 5, 7, $9,11,13^{*} 14,16,18,20,22 * 25 \mathrm{ML}$. This layer count excluded the wetting layer. The odd-numbered layers are favored between 5 and $13 \mathrm{ML}$ while even-numbered layers are favored between 14 and $22 \mathrm{ML}$, which averages to a beating wavelength $\lambda_{\text {beat }}=9 \mathrm{ML}$. As we will discuss below, this RBBB growth mode is a manifestation of the QSE, and the even-odd crossover phenomenon is the result of a beating of the interlayer spacing $d_{0}=2.86 \AA$ and the Fermi wavelength.

For the $\mathrm{Ge}(111)$ substrate, Figure $4.7 \mathrm{~g}$ shows a smooth $5 \mathrm{ML}$ film of $\mathrm{Pb}$ on $\mathrm{Ge}(111)$ with $5 \mathrm{ML}$ deep voids. Upon subsequent deposition, the voids close before bilayer growth commences. The even-odd crossovers also occur at the same location as for $\mathrm{Si}(111)$, indicating that the smaller band gap for electron confinement on Ge does not alter the bilayer growth and stability crossovers of the films.

In $\mathrm{Pb}(111)$ thin films, the $\lambda_{\mathrm{F}} / 2$ oscillations interfere with the periodicity of the atomic layer spacing along the growth direction so that the (possible) oscillation pattern in the thin film stability is determined by the relation between $\lambda_{\mathrm{F}}$ and atomic layer spacing $d_{0}[8,9] . \mathrm{Pb}(111)$ films are particularly interesting in this regard because each bilayer increment of the film thickness accommodates three additional antinodes of the Fermi wavefunction or $3 \lambda_{\mathrm{F}} / 2 \approx 2 \mathrm{ML}$. This accidental near commensurability causes quasi-bilayer oscillations in the thin film stability.

Besides the $\mathrm{Pb}$ growth on $\mathrm{Si}$ substrate, $\mathrm{Pb}$ growth on $\mathrm{Cu}(111)$ substrates has also been studied [35]. Three-dimensional islands of various heights were grown on the $\mathrm{Cu}$ surface at the temperature of $300 \mathrm{~K}$ and above. The heights of $6,8,10-11$, and $15 \mathrm{ML}$ were found, but not of 5, 9, 12, or $16 \mathrm{ML}$.

\subsubsection{First-Principles Calculations}

In ab initio calculations, the surface energy of a $\mathrm{Pb}$ film is computed using the following formula:

$$
E_{\mathrm{s}}=\frac{1}{2}\left[E_{\text {film }}^{\mathrm{tot}}(n)-n E_{\mathrm{bulk}}\right]
$$


$E_{\text {film }}^{\text {tot }}(n)$ is the total energy of the film with $n$ layers and $E_{\text {bulk }}$ is the total energy per layer of the bulk. As a result, these total energies should increase linearly with the film thickness modulated by some small fluctuations. By fitting the data with a linear function and subtracting the linear part from the total energies, the desired surface energies $E_{\mathrm{s}}$ can be obtained.

Shown in Fig. 4.8 are the surface energies of freestanding $\mathrm{Pb}$ films and their discrete second derivatives. The surface energy oscillates mostly with film thickness in a bilayer fashion. However, this bilayer pattern is interrupted at least twice by crossovers at films of 8 layers and 17 layers, as seen in Fig. 4.8. The separation between the neighboring crossovers is nine monolayers. Clearly, the oscillation with film thickness persists for films as thick as 26 monolayers. From Fig. 4.8b, the films of $4,6,9,11,13,15,18,20$, and 22 layers are stable. These results agree well with experimental investigations [8].
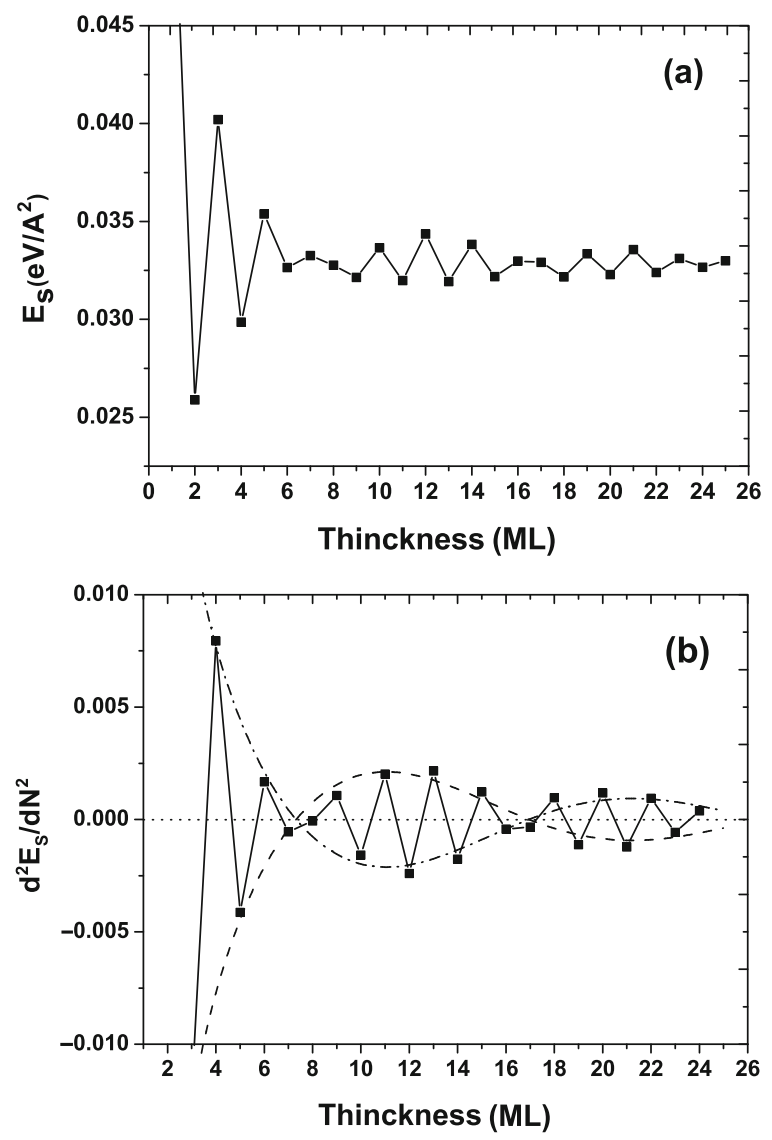

Fig. 4.8 (a) Surface energies of freestanding Pb films. (b) The discrete second derivative of surface energy of freestanding $\mathrm{Pb}$ films 
For $\mathrm{Pb}$ films on the $\mathrm{Ge}(111)$ substrate, there is a $11 \%$ mismatch between the lattice constants (Ge, 5.76 $\mathrm{Ab}, 5.04 \AA$ ). This large mismatch makes it impossible to carry out directly first-principles calculations for the $\mathrm{Pb}(111)$ and $\mathrm{Ge}(111)$ interfaces. A technical trick to overcome this obstacle is to rotate the substrate by $30^{\circ}$ and place a $2 \times 2 \mathrm{~Pb}$ unit cell on a bulk truncated $\mathrm{Ge}(111)-\left(\sqrt{3} \times \sqrt{3} R 30^{\circ}\right)$ surface. Such a method enables to accommodate the lattice mismatch between $\mathrm{Ge}$ and $\mathrm{Pb}$ and the substrate remains semiconducting. Another way adopted is to artificially compress the substrate to match the lattice constants, such as for $\mathrm{Si}(111)$ substrate in [35]. But in this approach, the substrate may become metallic and change its conducting nature, which is not desirable.

The surface energies of $\mathrm{Pb}$ films on the $\mathrm{Ge}(111)$ substrate and their discrete second derivatives are plotted in Fig. 4.9. Similar to the freestanding films, there are bilayer oscillations interrupted by crossovers. The separation between the crossovers is about nine monolayers but the crossovers are located at 5 monolayers, 13 mono-
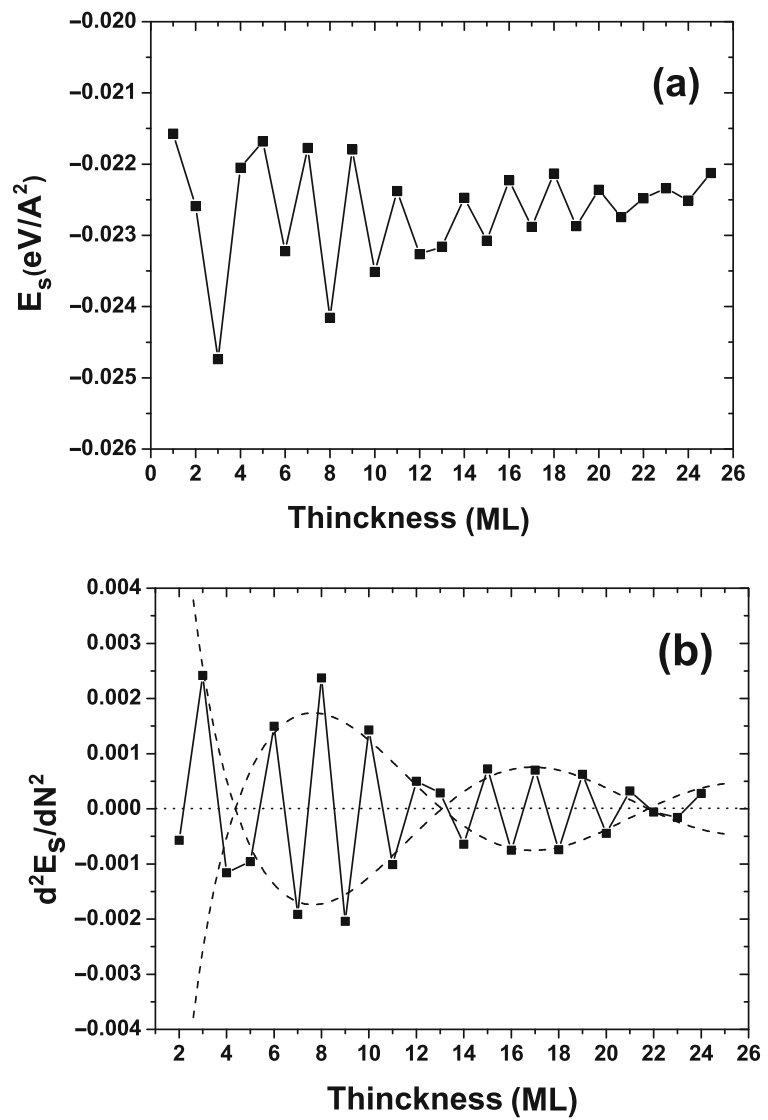

Fig. 4.9 (a) Surface energies of $\mathrm{Pb}(111)$ films on a $\mathrm{Ge}(111)$ substrate. (b) The second derivative of surface energy of $\mathrm{Pb}(111)$ films on a $\mathrm{Ge}(111)$ substrate 
layers, and 23 monolayers, which are different from the freestanding case. From Fig. 4.9b it can be inferred that the films are stable at 3, 6, 8, 10, 12, 15, 17, 19, and 21 layers. This agrees almost perfectly with a recent experiment of $\mathrm{Pb}$ film growth on the $\mathrm{Ge}(111)$ substrate [8]. This indicates that our first-principles calculations have captured the essential physics.

\subsubsection{Band Structure and Beating Phenomena of Pb(111) Films}

To understand the magic stability of the $\mathrm{Pb}$ films, including the unique growth behaviors discussed above, the band structure of $\mathrm{Pb}$ films with different thickness is shown in Fig. 4.10. For s-p metals, the QWS is often described by (4.1). For a film with a thickness of $N$ monolayer and interlayer spacing $d$ (4.1) becomes

$$
2 k N d+2 \phi_{\mathrm{s}}=2 n \pi
$$

Using (4.6), one can calculate the periodicity for the QW states crossing the Fermi level, $\Delta N=\frac{\pi}{k_{\mathrm{F}} d}$, where $k_{\mathrm{F}}$ is the Fermi wave vector. We use the value of $k_{\mathrm{F}}^{\prime}=1.596 \dot{\mathrm{A}}$, which was obtained from the de Hass-van Alphen measurement $[36,37]$. Since the energy band comes from band folding from the second Brillouin zone, we have $k_{\mathrm{F}}=2 k_{\mathrm{BZ}}-k_{\mathrm{F}}^{\prime}=0.61 \mathrm{~A}^{-1}$ and $\Delta N=1.8 \mathrm{ML}$, indicating the QWS crossing the Fermi level for every incremental increase in the film thickness of about 1.8 layers, which is consistent with the experimental finding of bilayer stability.

There exists a small difference between the $2 k_{\mathrm{F}}$ and the $k_{\mathrm{BZ}}$, and this difference will accumulate as the film grows thicker, leading to the "beating effect." The beating period of the crossovers is then given by

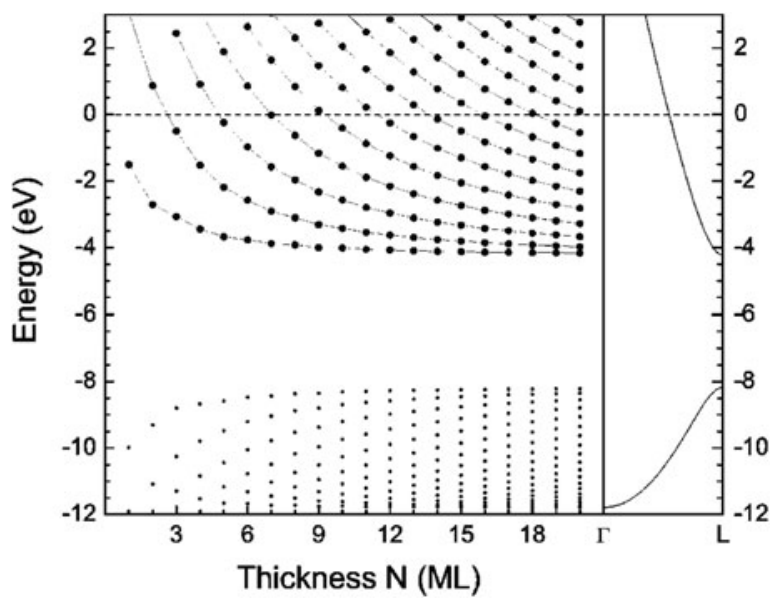

Fig. 4.10 Quantum well state energies as a function of $\mathrm{Pb}(111)$ layer thickness calculated at $\bar{\Gamma}$ using the local density approximation to density functional theory. The bulk energy dispersion is also shown. The horizontal dashed line indicates the Fermi level. Reproduced from [6] 


$$
\lambda_{\text {beat }}=\frac{\pi}{2 k_{\mathrm{F}}-k_{\mathrm{BZ}}} \approx 9 \mathrm{ML}
$$

The salient features of the re-entrant bilayer-by-bilayer mode are attributed to the quantum nature of the film stability, as confirmed quantitatively in DFT calculations disscussed above.

\subsubsection{Transition from Smooth Film to Island Regime}

The island morphologies can never be formulated as a unique, reversible, and pathindependent function of deposition temperature, annealing temperature, and deposition amount, even though 2D flat-top objects do form at low deposition temperatures because of size quantization effects. The contact area between the islands and the substrate changes upon annealing whereas for a continuous film, the contact area remains preserved during the morphological evolution. Reduction of the surface energy upon creating taller islands should ultimately lead to 3D clusters in thermodynamic equilibrium [38]. Hence, the stability arguments presented for the case of a closed 2D morphology cannot be used here. At low temperatures, on the other hand, one can think of a local equilibrium concept where a number of nearby local energy minima may be accessible within the "attempt range" of the system. From this perspective, the gradual evolution of taller islands can be thought of as successive jumps over multiple energy barriers, each time reaching a lower point in the global energy landscape.

The demarcation between the low temperature quantum growth region and the high temperature thermodynamic limit is a delicate one. For the quantum regime, the local stability concept is better suited whereas at high temperature the global energy landscape must be used [21]. It is well known that deposition of $\mathrm{Pb}(111)$ at $150-220 \mathrm{~K}$ produces flat-top islands with fairly uniform heights [25]. The selected height can be tuned by changing the deposition or post-annealing temperature: the lower the temperature, the lower the average island height. The first selected height turns out to be $5 \mathrm{ML}$, then comes the 7 and $9 \mathrm{ML}$ islands at elevated temperatures. Once the 5 (or 7 or $9 \mathrm{ML}$ ) high islands cover the whole surface, the growth continues in layer-by-layer or bilayer-by-bilayer fashion depending on the temperature [8]. This new growth mode, i.e., the initial formation of flat-top islands followed by $2 \mathrm{D}$ layer growth, has been explained in terms of quantum phase separation [22].

The roughening of initially flat continuous films (grown at very low temperature) upon warming is closely connected to this quantum phase separation: films deposited at 110-150 K exhibit some surface roughness because of the limited surface diffusion at low temperature. As the temperature is raised, the film first becomes smooth and then upon further annealing it will develop deep holes that go all the way down to the interfacial wetting layer (see Fig. 4.11). The ejected material from these holes accumulates around the holes, thus creating local areas of higher film thicknesses. Figure 4.11 shows the evolution of an initially 6.5 ML thick Pb deposit (wetting layer $+5.5 \mathrm{ML}$ ) on a $\operatorname{Si}(111) 7 \times 7$ substrate, as seen with STM. Snapshots 


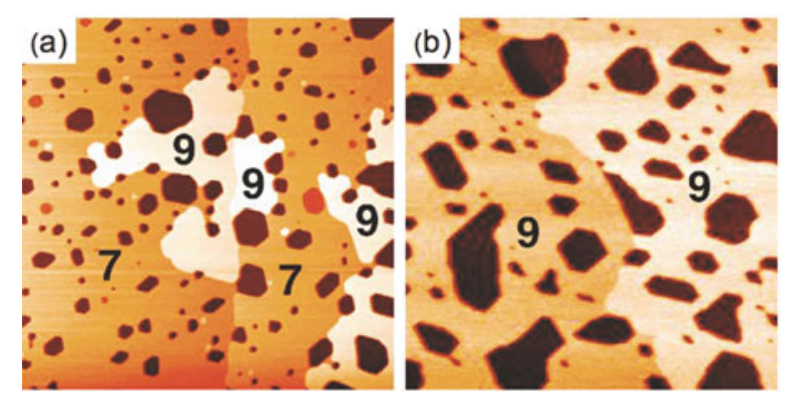

Fig. 4.11 $500 \times 500 \mathrm{~nm}^{2}$ images showing the evolution of a predominantly $7 \mathrm{ML}$ film into a $9 \mathrm{ML}$ film upon thermal annealing. The nominal coverage is fixed. The initial deposition of 6.5 ML was done at $150 \mathrm{~K}$. Both images show a substrate terrace passing through middle of the frames. (a) Image recorded at $225 \mathrm{~K}$ during the evolution. The 7 and $9 \mathrm{ML}$ tall areas are indicated. Very few $5 \mathrm{ML}$ speckles can be seen. (b) At $250 \mathrm{~K}$, all the holes are $9 \mathrm{ML}$ deep; the 5 and $7 \mathrm{ML}$ tall areas no longer exist

were taken as the film thickness evolves from 7 to $9 \mathrm{ML}$. These thicknesses are measured from the wetting layer. While the $7 \mathrm{ML}$ thick film evolves into a 9ML thick film at $225 \mathrm{~K}$, see Fig. 4.11a, the 9 ML film is still stable at $250 \mathrm{~K}$ (Fig. 4.11b). Also, it is known that the $5 \mathrm{ML}$ film is significantly unstable compared to the $7 \mathrm{ML}$ film on $\operatorname{Si}(111) 7 \times 7$ in close resemblance to reference [21]. In Fig. 4.11a, only a few small $5 \mathrm{ML}$ thick speckles are left whereas they have totally disappeared in Fig. 4.11b. Although the films shown in these images are not closed, the enormous lateral size of the connected pieces validates the use of thin film stability arguments. The general trend is that thinner films break up at lower temperature as compared to the thicker films.

The above picture emphasizes once more the irreversible approach toward thermodynamic equilibrium, starting from quantum growth regime. This transition is a very complex issue and at this point we can only speculate as to how this might happen, as we will do in the following paragraphs. In this context, one report particularly caught our attention [39]: the authors of this study deposited $11 \mathrm{ML} \mathrm{Pb}$ onto the $\mathrm{Si}(111)(\sqrt{3} \times \sqrt{3}) R 30^{\circ}-\mathrm{Pb}$ surface (which can be viewed as a bulk truncated $\mathrm{Si}(111)$ surface terminated with $1 / 3 \mathrm{ML}$ of $\mathrm{Pb}$ ) at $110 \mathrm{~K}$ and monitored the film morphology with X-ray diffraction as they increased the sample temperature. The authors reported that at some particular temperature $(280 \mathrm{~K})$ the film reaches a particular local equilibrium at which the discrete second derivative of the thickness distribution, obtained from the X-ray data, correlates with the thickness-dependent surface energy. Figure 4.12 is replicated from this work and shows the fractional surface area covered with $N$ monolayers. Based on the above discussion, the presence of a thickness less than 11 ML should not be expected, nonetheless their weight in the spectrum is significant. However, the statistical analysis given in this work, which correlates the height distribution to the surface energy, may be questionable because the authors used a local equilibrium (low temperature) argument at high temperature where mass transport takes place over several hundreds of nanometers. 


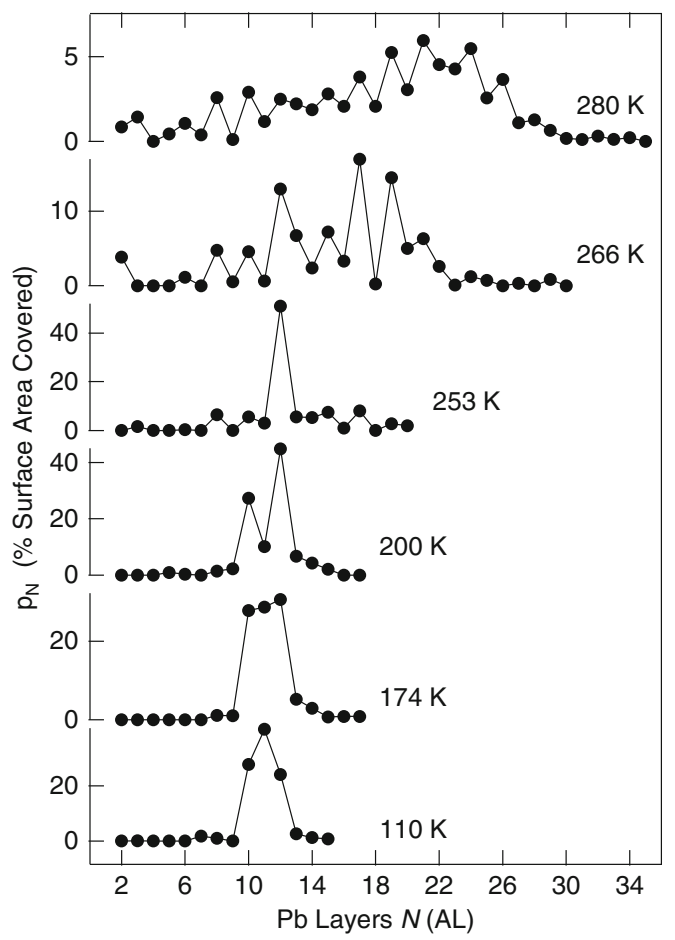

Fig. 4.12 Evolution of the $\mathrm{Pb}$ thickness distribution as a function of annealing temperature. Initial low temperature deposition of $11 \mathrm{ML} \mathrm{Pb}$ produces a narrow width distribution. The thickness distribution broadens at elevated temperatures [39]

Instead, it can be argued that one should consider the global energy landscape which includes the surface free energy of the island facets and that of the exposed wetting layer. Thus, one probably cannot expect to find a particular temperature where the calculated surface energy and experimental thickness distribution correlate over a nearly $30 \mathrm{ML}$ wide thickness window. The explicit assumption that each particular film thickness is in local equilibrium with its neighboring thicknesses $N \pm 1$ may not be valid. This would imply that, e.g., 5-6-7 ML and 19-20-21 ML thick areas maintain their local equilibrium at the same temperature. However, the STM data indicate that once the tall 19-20-21 ML mesas come into being, the 5-6-7 ML thick areas are no longer in local equilibrium and do not exist. Instead, the statistical analysis should have indicated a trend toward 3D cluster formation.

\subsection{Role of Friedel Oscillations in Thin Film Stability}

So far QSE-related oscillations in film stability are reasonably well understood, some quantitatively, some still at a qualitative level. One particular question is why QSEs are so robust in $\mathrm{Pb}(111)$ films, affecting a thickness range more than $20 \mathrm{ML}$ 
in both experiments and first-principles calculations. Also, why is it not that robust in other crystal orientations of $\mathrm{Pb}$, in $\mathrm{Cu}$ and $\mathrm{Al}$ films?

Vacuum and substrate interfaces at the thin film boundaries introduce steep discontinuity of the positive background charge. The collective screening response of the electron gas against this sharp discontinuity creates the well-known Friedel oscillations [18]. Nearly free electron calculations considered before have been carried out against a constant background and completely ignore the presence of the Friedel oscillations [27]. The wavelength of these oscillations is $\lambda_{F} / 2$, which is incidentally the same key parameter in the free electron QSE. So, it is far from obvious how to extract the role of the Friedel oscillations in QSE .

To be more specific, according to free electron calculations, QSE should be washed out beyond 10-15 ML [27]. On the other hand, both experiments and firstprinciples calculations suggest that stability oscillations of the $\mathrm{Pb}(111)$ films last up to $25 \mathrm{ML}$ or more [8]. The reason for this robustness of the apparent quantum size effect has remained a mystery for a very long time.

Conventionally Friedel oscillations decay according to $1 / z^{2}$, where $z$ is the depth measured from the interface. Recent DFT calculations [40] confirm this behavior for freestanding films of $\mathrm{Pb}(110), \mathrm{Pb}(100)$, and $\mathrm{Al}(111) \mathrm{Pb}(111)$ films, however, exhibit an unusual $1 / z$ decay law. This guarantees the persistence of the $\lambda_{F} / 2$ charge corrugation up to $25 \mathrm{ML}$ or more. We also note that there are two sets of Friedel oscillations emanating from the two interfaces of the film. Direct evidence for the role of Friedel oscillations (at the DFT level) comes from the extraordinarily large lattice relaxation at the center of the film [40].

The $1 / z$ decay law is expected only for quasi-1D electron systems, or equivalently flat Fermi surface segments. Indeed, recent DFT calculations show convincingly such Fermi surface nesting features along the $\mathrm{Pb}[111]$ direction [35, 40, 41], an intriguing property which merits further exploration.

\subsection{Structural, Electronic, Physical, and Chemical Properties of Quantum Films}

Thorough study of QSE is essential in understanding the various physical and chemical properties of thin metal films of few atomic layers thick and other nanostructures of comparable size. Furthermore, this detailed understanding makes it possible to use the QSE as a tuning knob to adjust some of the physical and chemical properties of quantum systems. In the rest of this work, we will give a brief overview of the QSE on lattice relaxations, work functions, superconductivity, and chemical reactivity. We will also briefly describe how such properties can be tunned via metal alloying in the quantum regime.

\subsubsection{Lattice Relaxation}

Abrupt termination of the crysal at the vacuum interface causes the well-known Friedel oscillations of the electronic charge density with the period of $\lambda_{F} / 2$ or, 
equivalently, $2 k_{\mathrm{F}}$, perpendicular to the film [20]. That means, the charge density close to the surface does not share the same periodicty with the crystal along the direction normal to the film. Hence, one would expect the ion cores at the top few layers to be displaced slightly with respect to bulk equilibrium positions [22, 42, 43]. This behavior is observed as bilayer oscillations in the atomic layer spacing $d_{12}$ and $d_{23}$ in $\mathrm{Pb}(111)$ films via LEED-IV (intensity versus electron energy) studies [42]. There it was shown that for a few selected $\mathrm{Pb}$ thicknesses the first layer spacing $d_{12}$ (as counted from the surface) is contracted compared to its bulk value $\left(d_{12}\right.$ is negative) while the second layer spacing $d_{23}$ is slightly expanded. Actually, these results are very similar to the relaxation of the top few layers of bulk $\mathrm{Pb}$ as expected from Friedel oscillations [43, 44]. On the other hand, QSE might add a thicknessdependent variation in the layer spacing to this picture due to confinement. This effect, however, turns out to be quite small and cannot be detected by LEED method due to limited probing depth.

Much larger relaxations have been reported based on $\operatorname{HAS}[34,45,46]$ and STM [24] measurements, with values up to $-30 \%$ for $d_{12}$ and $+15 \%$ for $d_{23}$ around the bulk equilibrium spacing [45]. Due to the nature of these techniques, however, the observed relaxations should be attributed to the strong electronic charge density variations around the vacuum boundary of the quantum well instead of the actual ion core displacemets.

Floreano et al. [46] have measured the atomic layer relaxations inside thin films with X-ray diffraction and X-ray reflectivity methods and concluded that the outermost layer $d_{12}$ exhibits much smaller oscillations (about 5\%) compared to those given by HAS measurements. Large number of parameters used in the fitting of their rod scans introduce limited confidence on the results. Czoschke et al. [42] have reduced the number of the parameters employing a theroretical model. They have calculated the derivative of the charge density $\partial \rho / \partial z$ inside the film using free electron model. Assuming the lattice displacements should be proportional to this gradient, they have obtained a displacement pattern to which the X-ray reflectivity data is to be fitted. The result obtained in this fashion for a $10 \mathrm{ML} \mathrm{Pb}$ film on $\mathrm{Si}(111)$ $\sqrt{3} \times \sqrt{3} R 30^{\circ}-\mathrm{Pb}$ gave a $d_{12}$ contraction of $9 \%$ (a $5 \%$ contraction was found for a 9 ML film (Chiang, Private Communication)). So, this model ends up with smaller relaxations compared to HAS and STM measurements, although $9 \%$ relaxation is still quite large. Although this model is physically intuitive and seemingly relevant, it is probably too simplistic. Not only the charge density in the film is model dependent but also the linear response of the ion cores to the charge density gradient is presumably not valid for the large relaxations obtained.

DFT calculations of ultrathin $\mathrm{Pb}(111)$ slabs on a variety of substrates confirmed the oscillatory nature of the interlayer spacing due to the $2 k_{\mathrm{F}}$ modulation of the charge density propagating into the bulk, as well as the superimposed oscillations as a function of thickness [9]. Figure 4.13 shows the interlayer relaxations $\Delta d_{12}$ and $\Delta d_{23}$ as a function of the $\mathrm{Pb}$ film thickness on a $\mathrm{Ge}(111)$ substrate [9]. The interlayer relaxation in a film of $N$ monolayer is defined as $\Delta d_{m, m+1}=\frac{d_{m, m+1}-d_{0}}{d_{0}} \times 100$ ( $d_{m, m+1}$ is the interlayer spacing between the $m$ and the $m+1$ layers). The by now familiar oscillation pattern including the even-odd crossovers is clearly reproduced. DFT results show that the first interlayer spacing $d_{12}$ is contracted by about 5.5\% 


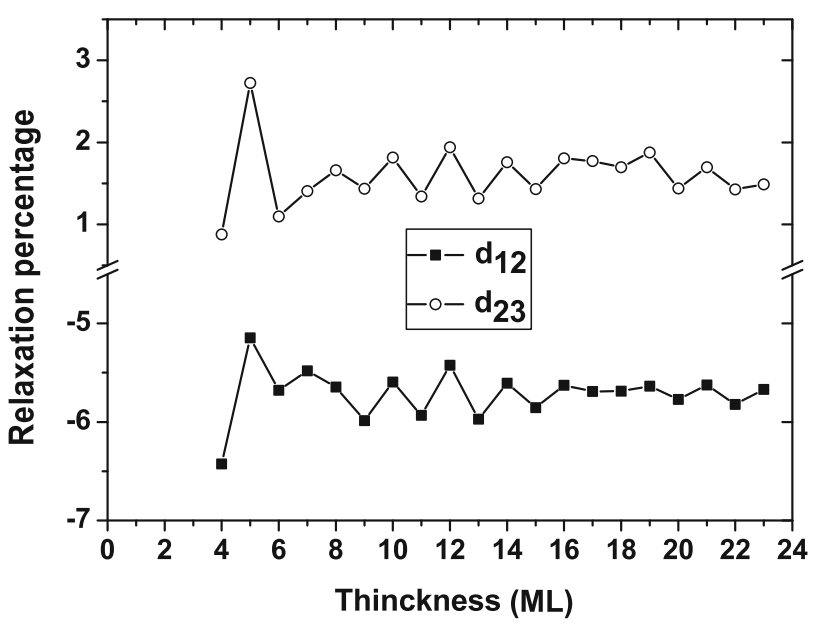

Fig. 4.13 Interlayer spacings $\Delta d_{12}$ and $\Delta d_{23}$ of $\mathrm{Pb}(111)$ films on $\mathrm{Ge}(111)$ substrate relative to bulk spacing. Reproduced from [9]

relative to the bulk value of the lattice constant while the second interlayer spacing $d_{23}$ is expanded by about $1.5 \%$. These results are in good agreement with the LEED measurements [47]. Note that the crossover positions of $\Delta d_{12}$ and $\Delta d_{23}$ are not the same as that of surface energy. Interestingly, the location of the even odd crossovers has shifted by a half beating period as compared to the even-odd crossovers in the corresponding surface energy $E_{\mathrm{s}}(L)$, suggesting that the lattice relaxation depends on the thickness according to $\mathrm{d} E_{\mathrm{s}}(L) / \mathrm{d} L$. This property is not yet fully understood.

\subsubsection{Work Function}

The work function (WF) is defined as the minimum energy required to remove an electron from the surface (see Fig. 4.14). The theoretical and experimental studies on the work function exhibit changes due to the alkali metal adsorption [48], crystallographic orientation [49], and the surface steps [50] and reveal that WF is related to the local density of states near the Fermi level. As we have discussed, the presence of QWS dramatically modulates the density of states at the Fermi level when the film thickness is varied. Therefore, one would expect to see thickness-dependent variations in the work function also.

Based on DFT calculations, Wei and Chou showed that, similar to the surface energy, the $\mathrm{WF}$ of freestanding $\mathrm{Pb}(111)$ films also reveals bilayer oscillations as a function of the film thickness [6]. Work function calculations for films on a $\mathrm{Ge}$ substrate have also been performed [9] and shown in Fig. 4.15. Bilayer oscillations are evident and even-odd crossovers exhibit $\sim 9 \mathrm{ML}$ periodicity similar to surface energy results. However, the location of the crossover points is phase shifted by half of a beating wavelength, similar to the behavior of the lattice relaxations. 


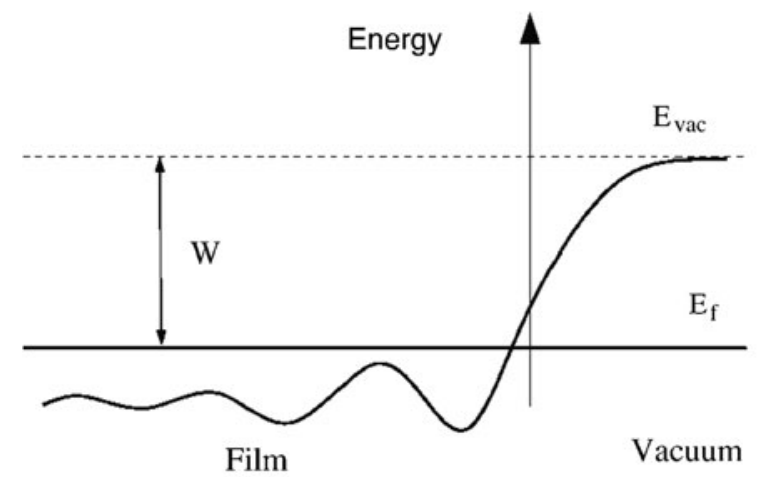

Fig. 4.14 Schematic drawing of energies at a film surface. The solid curve represents the electrostatic potential across the film surface. The oscillations in the potential are caused by the Friedel oscillations of the surface electron density. Its value deep in the vacuum side is usually called the vacuum energy

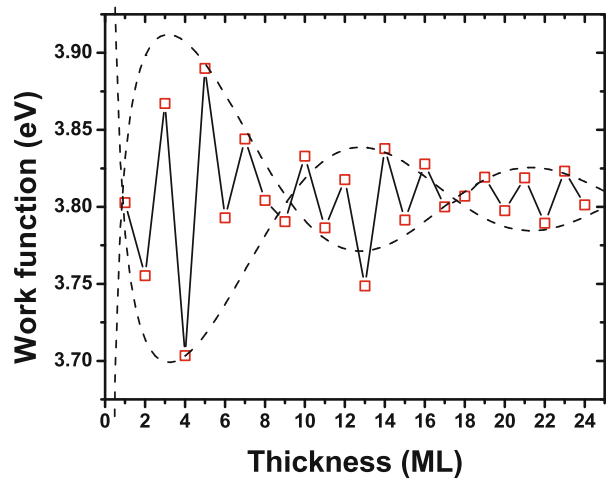

Fig. 4.15 The work function of $\mathrm{Pb}$ films as a function of thickness on $\mathrm{Ge}(111) \sqrt{3} \times \sqrt{3}$ substrate

Experimentally, WF oscillations have been measured with scanning tunneling spectroscopy. Qi et al. [51] have investigated the thickness dependence of the WF on flat-top-wedged $\mathrm{Pb}$ islands using the STM and STS measurements. Figure 4.16a shows the STM image of a flat-top Pb island with thickness increasing from left (11 ML) to right $(15 \mathrm{ML})$. The inset shows the atomically resolved STM image of the top (111) plane. Fig. 4.16b shows the cross-sectional profile along the line

Figure 4.16a, c, d shows the topographic and WF images of the island taken simultaneously. From the WF image, we can clearly see that even layers (12 and $14 \mathrm{ML}$ ) have larger work functions compared to odd layers (11, 13, and $15 \mathrm{ML})$. The tunneling current strongly depends on the tip-sample distance because of its dependence on the image potential. However, it can be shown that the quantity $\mathrm{d} \ln I / \mathrm{d} s$ is a constant to first approximation [52]. Hence, the work function can be determined from 


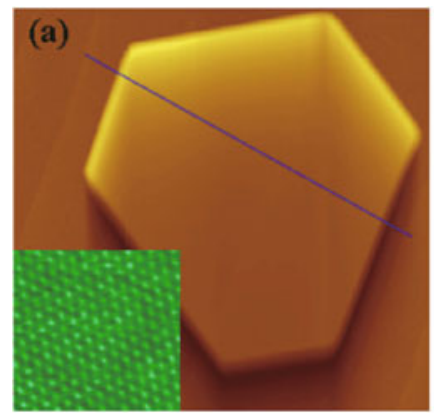

(b)
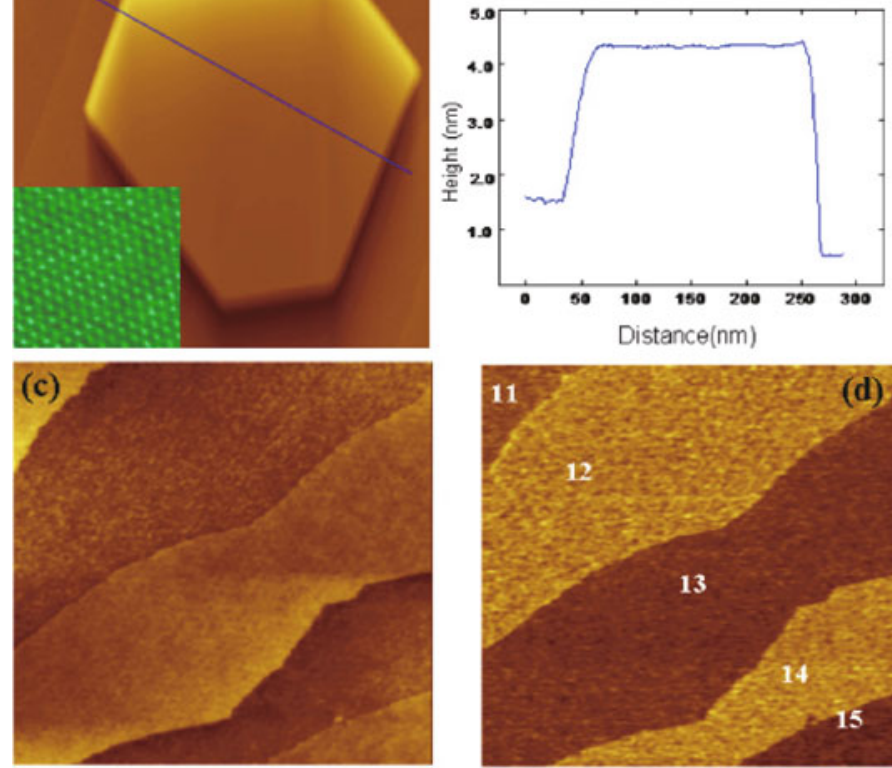

Distance $(\mathrm{nm})$

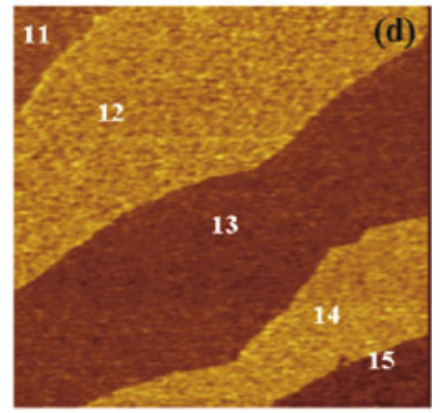

Fig. 4.16 (a) The STM image of a Pb island taken at $V=-0.8 \mathrm{~V}$ and $I=0.1 \mathrm{nA}$. Image size: $500 \times 500 \mathrm{~nm}^{2}$. Inset is a $5 \times 5 \mathrm{~nm}^{2}$ atomic resolution image $V=0.4 \mathrm{~V}, I=0.1 \mathrm{nA}$. (b) A line plot along the blue line in (a) to show the height profile. (c) The topographic and (d) local work function images taken simultaneously $V=-2 \mathrm{~V}, I=0.1 \mathrm{nA}$. The bright area has a larger work function than the dark area. Image size: $337 \times 337 \mathrm{~nm}^{2}$

$$
\phi \approx 0.95\left(\frac{\mathrm{d} \ln I}{\mathrm{~d} s}\right)^{2}
$$

and experimentally obtained via modulated tip sample distance.

Figure 4.17 shows the WF obtained by this method for a thickness range from 11 to $34 \mathrm{ML}$. As seen in this figure, the WF is larger for the even layers compared to the odd layers when $N$ is between 11 and $17 \mathrm{ML}$, whereas the reverse occurs when $N$ is between 19 and $26 \mathrm{ML}$ and so on. Overall, the bilayer oscillations of the WF are concomitant with an envelope function of beating at approximately 9 ML. The measured oscillatory periodicity and beating behavior show quite reasonable agreement with theoretical prediction [9].

Here we note that the similarity between the thickness dependences of the work function and the calculated lattice relaxation. They both exhibit even-odd crossover points at similar thicknesses. This behavior can be understood based on the charge spilling from the quantum well: higher the dipole moment because of the larger charge spill, higher the work function. This larger charge spill would also reduce the $d_{12}$ layer contraction as compared to the $d_{12}$ contraction of a bulk $\mathrm{Pb}(111)$ crystal. 


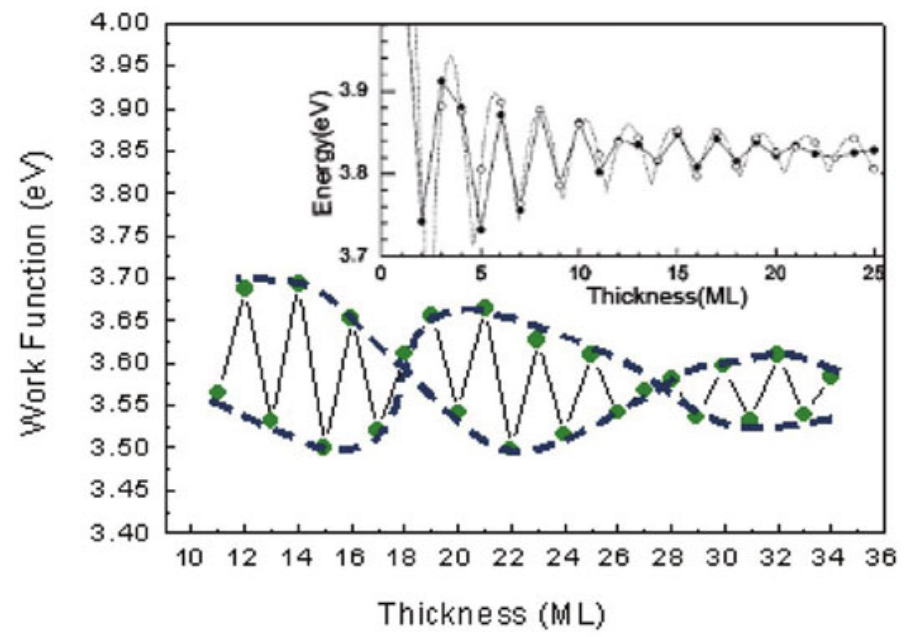

Fig. 4.17 Work function of $\mathrm{Pb}(111)$ films measured as a function of film thickness. Reproduced from [51]

So, the connection between work function and surface relaxation is established (Fig. 4.18).

Why the surface energy exhibits a different beating pattern compared to work function and lattice relaxations is not that transparent. According to Jellium calculations by Schulte, the charge spilling is minimum when a $2 \mathrm{D}$ subband is just touching the Fermi sphere and it exhibits a maximum when the Fermi level is located in the middle of the highest occupied and the lowest unoccupied subbands at $\Gamma$ [18]. This largest charge spilling case coincides with a local minimum of $E_{\mathrm{S}}(L)$. Therefore one tentatively would expect $E_{\mathrm{S}}(L)$ and work function to oscillate in phase. However, the Fermi energy also oscillates as well as the dipole strength. So, these two phenomena are entangled to each other and the physics of the beating would be revealed after a self-consistent treatment of the problem. Apparently, the result of such an analyses turns out to be a phase shift of about 4-5 ML, i.e., half of a beating wavelength.

Very recently, Miller, Chou, and Chiang presented an analytic derviation and numerical examples for the phase relations and the beating functions in terms of subband crossing of the Fermi level. Based on the standard quantum well model [33, $54,55]$, one can derive the following central equations for the chemical potential $\mu$ and surface energy per unit area $E_{\mathrm{s}}$ :

$$
\begin{aligned}
& \frac{1}{N^{2}} \frac{d}{\mathrm{~d} N}\left(N^{2} \mu\right)=\frac{3 \rho}{N D(\mu)} \\
& \frac{1}{N^{4}} \frac{d}{\mathrm{~d} N}\left(N^{4} E_{\mathrm{s}}\right)=\frac{3}{2} \rho\left(\mu-\mu_{\text {bulk }}\right)
\end{aligned}
$$



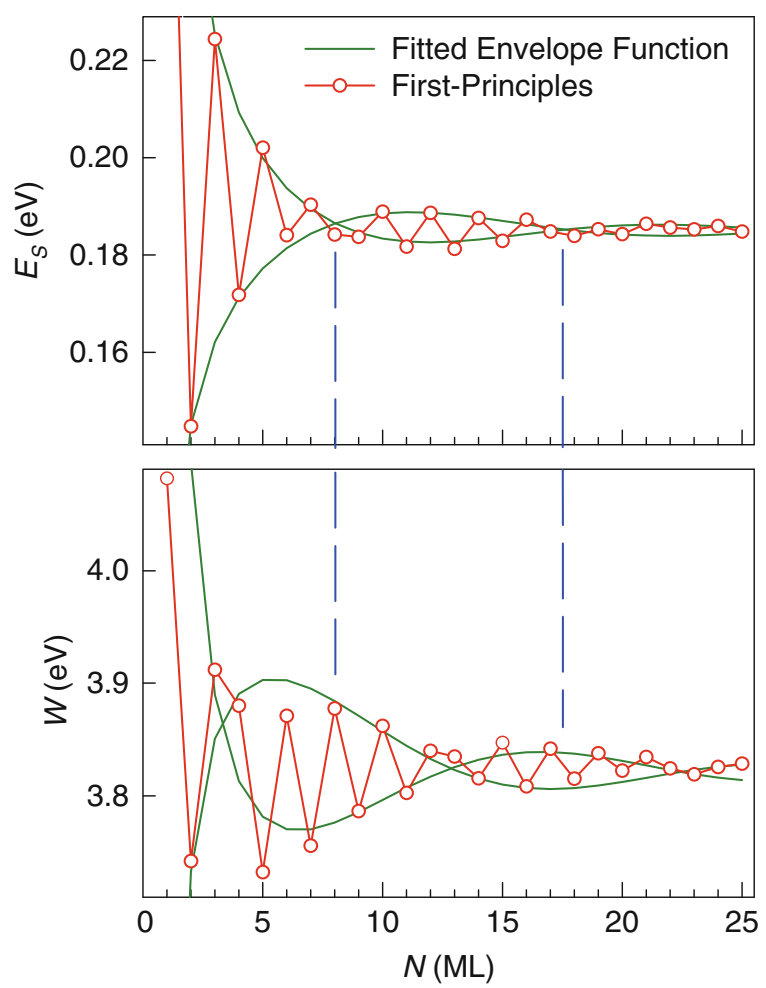

Fig. 4.18 Surface energy $E_{\mathrm{S}}$ per surface atom and work function $W$ as a function of thickness $N$ of freestanding $\mathrm{Pb}(111)$ films from a first-principles calculation. Also shown are envelope functions derived from a model fit to highlight the beating patterns. The two vertical lines are lined up with two adjacent nodes in $E_{\mathrm{S}}$ and highlight the out of phase relationship between the envelope functions of $E_{\mathrm{s}}$ and $W$. Reproduced from [53]

where $N, \rho$, and $D(\mu)$ are the film thickness, the electron density, and the density of states per unit volume at the chemical potential, respectively. $\mu_{\text {bulk }}$ is the bulk chemical potential. Equations (4.8) and (4.9) indicate that the rate of change of the chemical potential for increasing $N$ is related to the inverse of the density of states at the chemical potential, and the rate of change of the surface energy is related to the chemical potential.

The numerical results for $\mathrm{Pb}(111)$ are shown in Fig. 4.19. The top panel shows the evolution of the quantized electron structure $E_{n}$. The subbands cross $\mu$ with a period of $\Delta N=0.7 \mathrm{ML}$; the crossings are marked by the vertical dashed lines. The second panel shows $D(\mu)$. Each subband has a constant density of states, and the total density of states is a series of steps. Upon normalizing by the volume, it becomes a series of diminishing sawteeth. The third panel is $D(\mu)^{-1}$. The next panel displays the work function relative to the bulk limit: $\Delta W=-\Delta \mu \equiv \mu_{\text {bulk }}-\mu$. The bottom panel displays $E_{\mathrm{s}}$. Clearly, the oscillations in surface energy lead the oscillations in the work function by $1 / 4$ of a thickness period. 

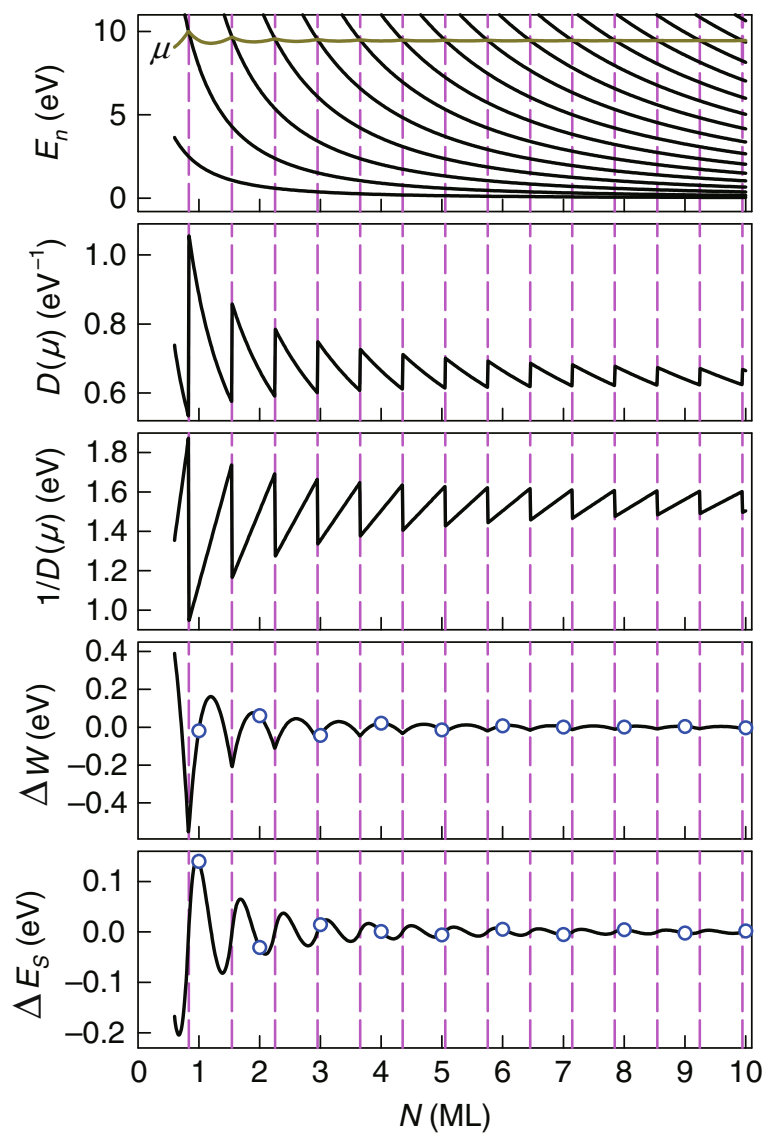

Fig. 4.19 The numerical results of surface energy and work functions from a quantum well model calculations. Reproduced from [53]

\subsubsection{Localized Surface Phonons and Electron-Phonon Interaction}

Electron-phonon coupling is an important factor that determines the superconductivity of a material. For a conventional superconductor such as $\mathrm{Pb}$, the effective electron-electron attraction necessary for the binding of cooper pairs is ultimately governed by electron-phonon coupling [56]. The formation of QWS strongly modifies the mechanical stability of the films, as reflected by the expansion and shrinkage of interlayer spacing [57]. Both of the factors speak directly to the possibility of modulating electron-phonon coupling and thus superconductivity of the $\mathrm{Pb}$ films.

Zhang et al. studied the interaction of electron-phonon coupling in $\mathrm{Pb}$ films with different thickness by using the variable temperature ARPES spectra and temperature-dependent PES [58]. The temperature-dependent PES for the 23 and 

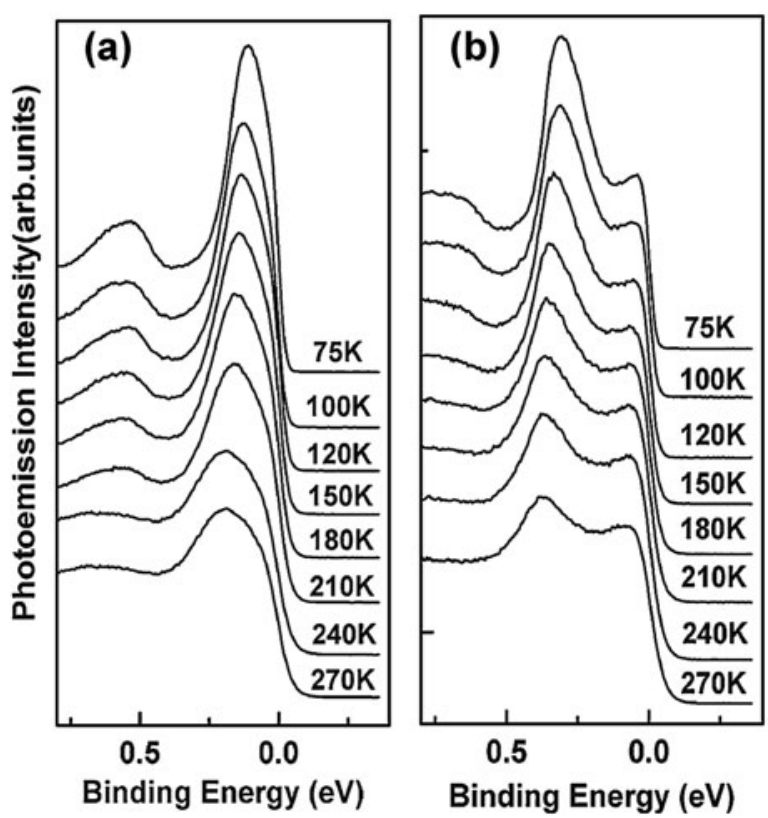

Fig. 4.20 Temperature-dependent photoemission spectra of $\mathrm{Pb}$ films for (a) $23 \mathrm{ML}$ and (b) $24 \mathrm{ML}$ collected within a temperature range of $75-270 \mathrm{~K}$. The vertical dashed lines reveal the variation of the QWS binding energy as the film temperature is changed [58]

$24 \mathrm{ML}$ films is shown in Fig. 4.20a and b. These results show that, with increasing substrate temperature, the peak position of the QWS shifts toward higher binding energies while the peak widths broaden. To find out the exact relation between the line width $(\Delta E)$ of the QWS and the temperature, a curve fitting by Voigt line shape was made. The fitting results are plotted in Fig. 4.21a for the films of 22 and 23 ML, where $\Delta E$ increases linearly with increasing temperature and exhibits prominent different slopes for the adjacent layers. The relation between this slope and the electron-phonon coupling strength, $\lambda$, is

$$
\lambda=\frac{1}{2 \pi k_{\mathrm{B}}} \frac{\mathrm{d} \Delta E}{\mathrm{~d} T}
$$

The $\lambda$ values derived from the QWS peaks for different thicknesses are shown in Fig. 4.21b (triangles), where only the values for stable layers $(15,17,19,21,22$, 23 , and $24 \mathrm{ML}$ ) are plotted. Besides an overall gradual increase of $\lambda$ toward the bulk value (1.55), an oscillation of $\lambda$ with a period of $2 \mathrm{ML}$ is clearly noted from 21 to $24 \mathrm{ML}$.

Theoretically, Yndurain and Jigato [59] recently do the DFT calculations to investigate the surface phonons and electron-phonon interaction. Zero parallel momentum phonon modes for $\mathrm{Pb}(111)$ freestanding slabs of widths ranging from 

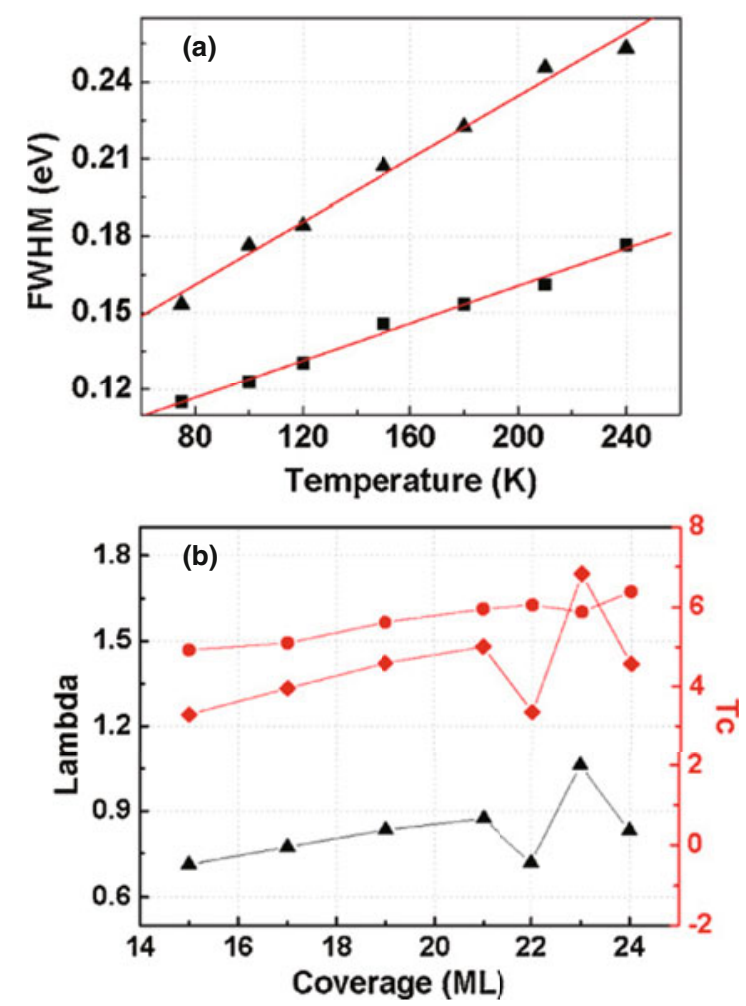

Fig. 4.21 (a) Lorentzian peak widths of the QWS of the $22 \mathrm{ML}$ (square) and 23 ML (triangle) films plotted as a function of temperature. (b) Measured $\lambda$ (triangles) and calculated superconductivity transition temperature (diamonds). Reproduced from [58]

3 to 14 layers are calculated and the results are shown in Fig. 4.22. From Fig. 4.22, one can see that both longitudinal and transverse surface modes take place at energies above the corresponding bulk values due to a $6 \%$ contraction of the surface layer (see Fig. 4.13). A bilayer oscillatory variation of both surface states is found, being more pronounced for the longitudinal modes than in the transverse ones as expected due to their different characters. It is expected that, like the surface energy, there should be one crossover among these layers with a beating period of $9 \mathrm{ML}$. However, the beating effect is not found in the DFT calculations. More theoretical studies are needed to reconfirm this.

\subsubsection{Thermal Expansion}

Temperature dependence of the PES spectra width was used to investigate manybody effects with regard to electron-phonon coupling. The peak positions, i.e., the binding energies of the QWS in the temperature-dependent spectra, could be used to understand the thermal expansion behavior of the thin films. From this dependence, 


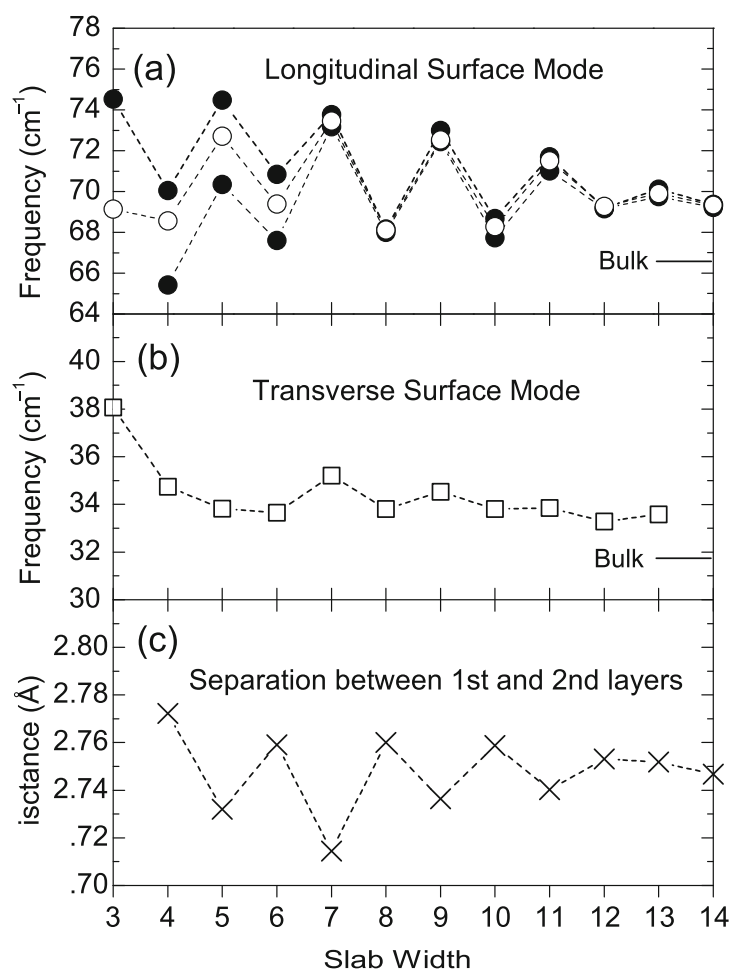

Fig. 4.22 Frequencies of localized surface phonon modes at $k_{\|}=0$ for different slab thicknesses. Panels (a) and (b) stand for longitudinal and transverse modes, respectively. In both cases the highest corresponding bulk mode frequency is indicated. In panel (a) the results considering the cases of both slab's ends free (bold symbols) and fixing one while leaving the other one free (open symbols) are shown. Panel (b) stands only for the case of one slab's ends fixed. Panel (c) shows the contraction of the outermost layer

one could deduce the thermal expansion coefficients of the $\mathrm{Pb}$ films of different thicknesses along the film normal direction.

Shown in Fig. 4.20 are the temperature-dependent photoemission spectra of $23 \mathrm{ML}$ (Fig. 4.20a) and 24 ML (Fig. 4.20b) Pb films by Zhang et al. [58]. From the spectra, the film morphology assumes an atomically flat surface over a macroscopic scale. Again, the strongest peaks correspond to the highest occupied QWS. Note that for both films including other films studied, the QWS peaks shift always toward higher binding energy with increasing substrate temperature. Furthermore, a dramatic difference between even and odd $\mathrm{Pb}$ layers can be clearly detected.

To quantify the results, the temperature dependence of the QWS binding energy for several $\mathrm{Pb}$ films is fitted linearly and shown in Fig. 4.23a. While the increased binding energy of the QWS in $\mathrm{Ag} / \mathrm{V}(100)$ system was explained as that the energy of the QWS decreases more than the Fermi energy of the substrate, it could be attributed to a different reason, namely the thermal broadening of the confinement 


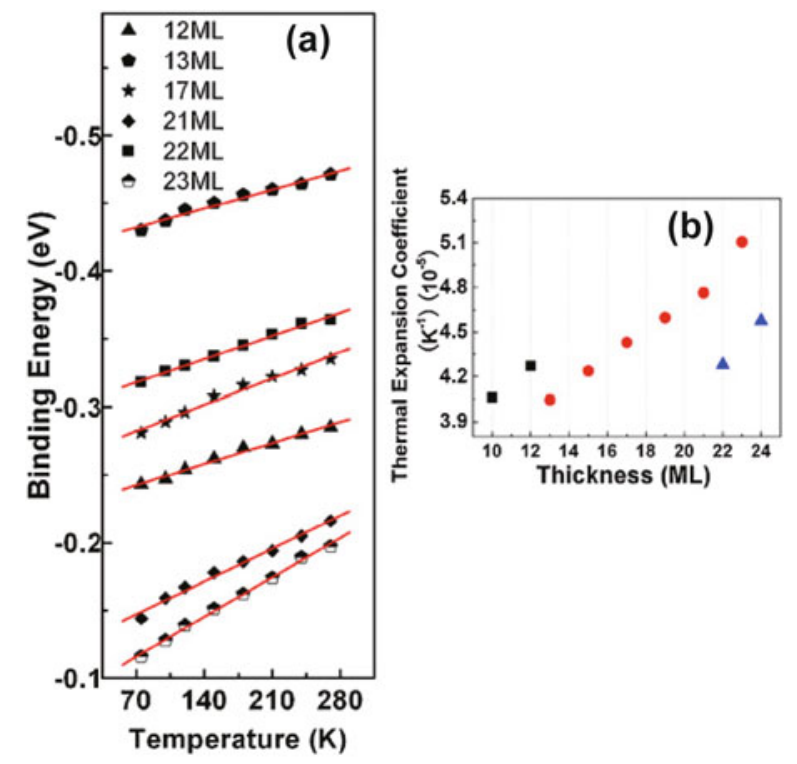

Fig. 4.23 (Color online) (a) Binding energy of the QWS plotted as a function of temperature. The dots and lines indicate the experimental data and linear fits, respectively. (b) Thermal expansion coeients of the $\mathrm{Pb}$ films along the confined direction calculated with the proposed model

well width, in addition to variation of the Fermi level of $\mathrm{Pb}$ films. For $\mathrm{Pb} / \mathrm{Si}(111)$, when temperature $(T)$ increases, $E_{\mathrm{F}}$ of the $\mathrm{Pb}$ film drops much more than $E_{\mathrm{F}}$ of $\mathrm{Si}$ does due to the dramatic difference in their thermal expansion coefficients, so there is a charge transfer from $\mathrm{Si}$ to the $\mathrm{Pb}$ film. These charges only stay at the $\mathrm{Pb}-\mathrm{Si}$ interface (no charge is expected in the $\mathrm{Pb}$ film), which shift up all energy levels equally of the $\mathrm{Pb}$ film until $E_{\mathrm{F}}(\mathrm{Pb})=E_{\mathrm{F}}(\mathrm{Si})$ is satisfied. Therefore, the Fermi energy of Si and its change have minor effect on the relative shift of the QWS in the $\mathrm{Pb}$ film. Within a simple free electron approximation, the temperature-dependence of the Fermi energy of a bulk material is described as

$$
\frac{\mathrm{d} E_{\mathrm{F}}}{\mathrm{d} T}=-2 E_{\mathrm{F}} \alpha_{\mathrm{r}}
$$

where $\alpha_{\mathrm{r}}$ is the linear thermal expansion coefficient of the material, and $E_{\mathrm{F}}$ is its Fermi energy relative to the bottom of valence band. For the thermal-induced shift of the substrate Fermi level, $\alpha_{\mathrm{r}}$ can be selected as $2.8 \times 10^{-6} \mathrm{~K}^{-1}$ (the bulk value). As for the thermal-induced shift of the QWS energy, such approximation should be reasonable regarding to the fact that the QWS energies of the $\mathrm{Pb}$ films involved are within a small energy window of $0.6 \mathrm{eV}$ below the Fermi level, which has been successfully used to determine the $\mathrm{Pb}$ band structure. The thermal-induced shift of the QWS energy ( $E_{\mathrm{QW}}$ ) with respect to the bottom of valence band has the following form where $\alpha_{Z}$ is the linear expansion coefficient along the confined direction: 


$$
\mathrm{d} E_{\mathrm{QW}} / \mathrm{d} T=-2 E_{\mathrm{QW}} \alpha_{\mathrm{Z}}
$$

The linear thermal expansion coefficients of the $\mathrm{Pb}$ films in the confined direction were calculated by the experimental thermal shift of QWS binding energy in terms of (4.11) and (4.12), and the results are shown in Fig. 4.23b. Several observations can be made as follows: (1) the thermal expansion coefficients in the film normal direction are greatly enhanced compared to the bulk $\mathrm{Pb}$; (2) there is a $2 \mathrm{ML}$ oscillation for the $\mathrm{Pb}$ films from 21 to $24 \mathrm{ML}$; (3) the overall trend is that a lower expansion coefficient corresponds to a film with a higher QWS binding energy. Since the thickness of the $\mathrm{Pb}$ films is very small, the linear expansion coefficient in the film plane ( $x y$-plane) should be very closed to that of bulk $\mathrm{Si}$, while in $z$-direction, a great enhancement can be expected because of what called Poisson effects and that the linear expansion coefficient of $\mathrm{Si}\left(2.8 \times 10^{-6} \mathrm{~K}^{-1}\right)$ is one order of magnitude lower than that of $\mathrm{Pb}\left(2.89 \times 10^{-5} \mathrm{~K}^{-1}\right)$. To understand the global enhancement of the linear thermal expansion coefficient along the confined direction, we use $\alpha_{\mathrm{r}}, \alpha_{\mathrm{P}}$, and $\alpha_{Z}$ to describe the linear expansion coefficient of the freestanding film, the confined film in the film plane, and the film normal $(z)$ direction, respectively. $\alpha_{Z}$ can be expressed as

$$
\alpha_{\mathrm{Z}}=\alpha_{\mathrm{r}}+\frac{2\left(\alpha_{\mathrm{r}}-\alpha_{\mathrm{P}}\right) \eta}{1-\eta}
$$

where $\eta$ is the Poisson ratio. Substituting $\alpha_{\mathrm{r}}$ and $\alpha_{\mathrm{P}}$ by the linear expansion coefficients of bulk $\mathrm{Pb}$ and bulk $\mathrm{Si}$, respectively, and taking $\eta$ as the Poisson ratio of bulk $\mathrm{Pb}(0.44)$, we obtain $\alpha_{z}$ that equals $2.414 \alpha_{\mathrm{r}}$. That means, in an ideal case, the thermal expansion in $z$-direction should be enhanced by 2.414 times of the bulk value, which is adequate to explain the global enhancement of the experimental data in $\mathrm{Pb} / \mathrm{Si}(111)$.

\subsubsection{Superconductivity}

Advances in the thin film growth via QSE also prove to be essential to study the superconductivity in 2D geometry. Because the normal state resistance is a critical factor in destruction of the superconductivity, excellent film quality is required to investigate the inherent physics of low-dimensional superconductors [60]. For example, when the normal state resistance reaches the quantum resistance for the Cooper pairs, the superconductivity disappears [61]. While the normal state resistance was the control knob in the earlier studies of the thin film superconductivity, atomically smooth films achieved in the quantum growth regime render the film thickness as the well-controlled fundamental parameter [12-14]. Indeed, it is already known that the boundary scattering sets the limit of the resistivity at low temperatures $[62,63]$.

Various groups have measured the superconducting transition temperature $T_{\mathrm{C}}$ of $\mathrm{Pb}$ films and $\mathrm{Pb}$ islands employing various methods such as contactless mag- 
netization [14], DC transport [12], and superconducting gap measurements [13]. Macroscopic coherence is obtained from the first two methods (Fig. 4.24), whereas the gap measurements give the local character of the superconductivity (Fig. 4.25).

Ozer et al. [14, 15] employed the superconducting quantum interference device (SQUID) to measure the superconductivity. This method eliminates the electrical contact issues because the measurements are performed inductively. On the other

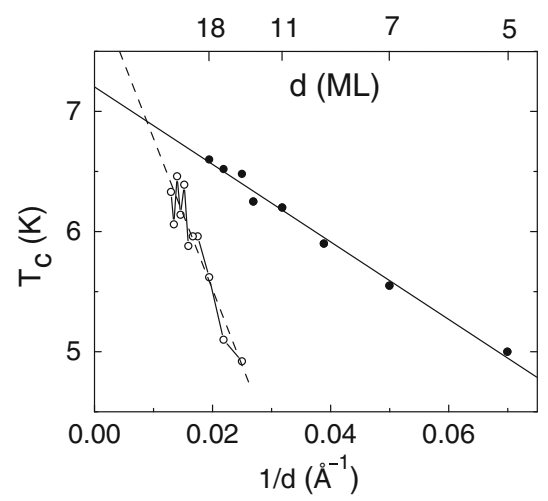

Fig. 4.24 $T_{\mathrm{C}}$ of $\mathrm{Pb}(111)$ films as a function of inverse film thickness. Filled dots show the magnetic measurements [14]; open circles correspond to transport measurements [12]

(a)

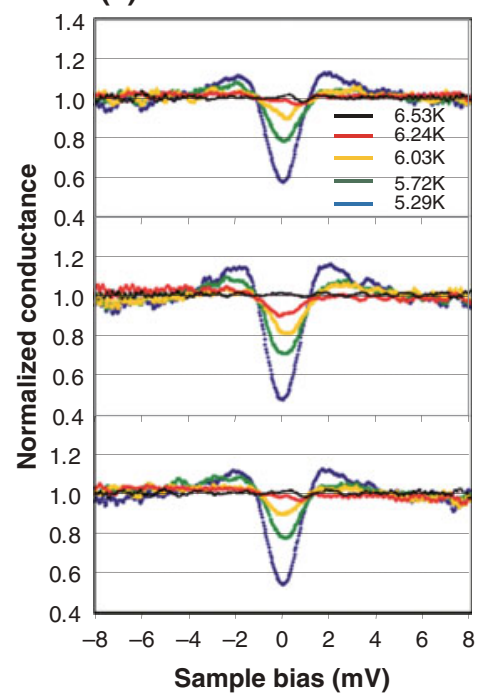

(b)

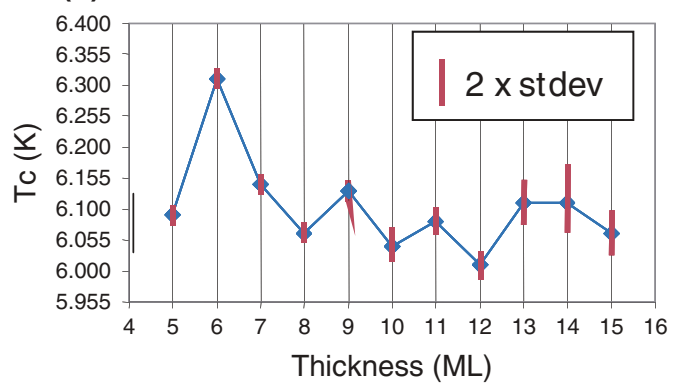

Fig. 4.25 $T_{\mathrm{C}}$ measurements from the opening of the superconducting gaps in scanning tunneling spectroscopy. (a) Gap of 5, 6, and $7 \mathrm{ML}$ thick $\mathrm{Pb}$ films at various temperatures. (b) $T_{\mathrm{C}} \mathrm{s}$ obtained from the gap measurements in panel (a). Reproduced from [13] 
hand, these measurements have to be carried out ex situ. This requires a protective cap layer to be deposited, which potentially can affect the superconducting properties. Also, in order to pick up a measurable magnetic signal, the macroscopic coherence must be established over all sample. DC transport measurements, however, detect the onset of the superconductivity via establishement of a single filamentary supercurrent.

Magnetic data in Fig. 4.24 exhibit a clear $1 / d$ dependence of $T_{\mathrm{C}}$, which can be explained in terms of the suppressed order parameter close to the film boundaries according to Simonin's theory [64]. Possible bilayer $T_{\mathrm{C}}$ oscillations cannot be seen in this data set because only the stable thicknesses dictated by QSE and separated by $2 \mathrm{ML}$ have been prepared. $T_{\mathrm{C}}$ values extrapolate to $7.2 \mathrm{~K}$ in the bulk limit which is the true bulk value. Same $1 / d$ thickness dependence of $T_{\mathrm{C}}$ is observed also for $\mathrm{Pb}-\mathrm{Bi}$ alloys films. $\mathrm{Pb}_{89} \mathrm{Bi}_{11}$ and $\mathrm{Pb}_{80} \mathrm{Bi}_{20}$ films exhibit extrapolated bulk $T_{\mathrm{C}}$ values of 7.69 and $8.05 \mathrm{~K}$, respectively $[31,65]$. Same figure also shows the transport measurements with monolayer resolution indicating that most of these films were grown in the classical layer-by-layer growth regime. In this data set, bilayer oscillations of $T_{\mathrm{C}}$ are evident and it can be explained by oscillating density of states and/or electron-phonon coupling due to QSE [12].

We note that the magnetic and transport data show significantly different slopes in these $T_{\mathrm{C}}$ versus $1 / d$ plots. Only the magnetic data extrapolate to the correct bulk value. Metalic Au protective layer employed for transport data may cause this difference because of a possible proximity effect. Note that amorphous Ge layer was used to protect the samples prepared for magnetic measurements. In situ superconducting gap measurements [13] shown in Fig. 4.25 exhibit another contrast quite different in character: this time $T_{\mathrm{C}}$ does not exhibit a significant thickness dependence and bilayer oscillations depicted in the figure are much smaller than those observed in transport measurements. This radically different behaviors may be due to the fact that magnetic and transport measurements rely on the establishement of the macroscopic coherence, i.e., they are sensitive to both amplitude and phase of the order parameter. On the other hand, local scanning tuneling spectroscopy (STS) measurements detect only the amplitude of the order parameter.

A recent study reported superconducting gap opening in $2 \mathrm{ML}$ thick $\mathrm{Pb}$ films $[66,67]$. This is interesting because in a $2 \mathrm{ML} \mathrm{Pb}$ film, only one $2 \mathrm{D}$ subband is allowed to exist due to the relation $3 \lambda_{\mathrm{F}} / 2 \approx 2 \mathrm{ML}$. Also, this work reports two different $T_{C}$ values for 2 ML films residing on phase separated $\sqrt{3} \times \sqrt{3}$ and $1 \times 1$ substrate reconstructions. This last point shows the effect of the interface on the thin film superconductivity.

Film morphology obtained by quantum growth also allows the incorporation of nanostructures into the picture providing a control knob of some superconducting parameters [15]. STM images of $9 \mathrm{ML}$ thick $\mathrm{Pb}$ films are shown in Fig. 4.15. In these images, slight overdose of $\mathrm{Pb}$ causes $2 \mathrm{ML}$ tall mesas (a) and slight underdose of $\mathrm{Pb}$ creates $2 \mathrm{ML}$ deep voids (b) as dictated by QSE (see Sect. 4.4.1). Pb is a type I supeconductor in bulk form; however, it becomes type II character below a crtitical thickness of $\sim 250 \mathrm{~nm}$ [68]. As a result, magnetization loops show strong hysteresis as shown in Fig. 4.15c, d. Wide-open loops, belonging to the films with 

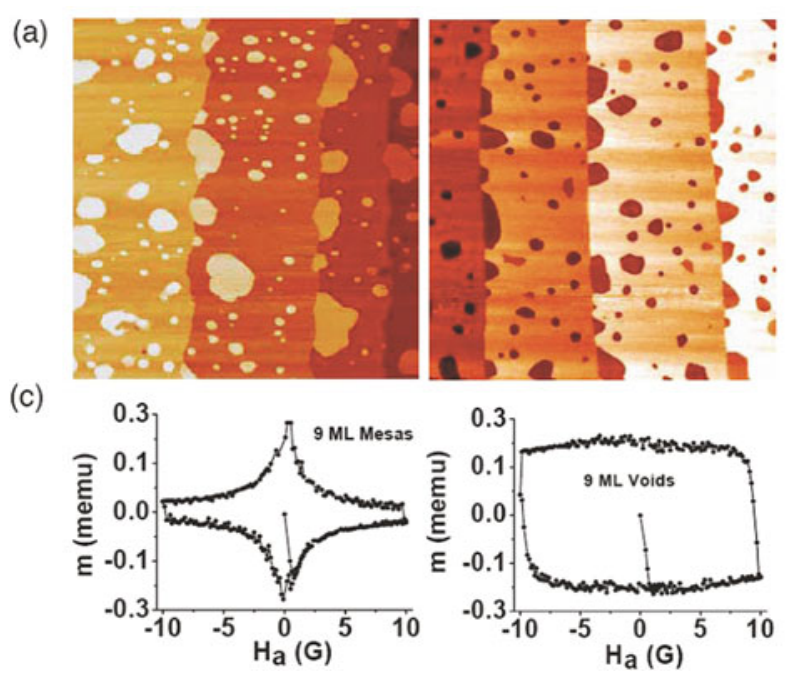

(d)

Fig. 4.26 $700 \times 700 \mathrm{~nm}^{2}$ STM images of Nano-patterned 9 ML Pb(111) films and the corresponding hysteresis loops. (a) Film with 2 ML tall mesas, (b) film with 2 ML deep voids, (c) hysteresis loop of film with mesas, and (d) hysteresis loop of film with voids. For both samples, the lateral area is $3 \times 3 \mathrm{~mm}^{2}$ and the magnetic data are taken at $2 \mathrm{~K}$. Reproduced from [15]

voids, are due to strong flux pinning inside these blind holes and directly related to roboust superconducting current density, $J_{C}$, which reaches as high as $10 \%$ of the theoretical depairing density. Also, this reference gives a simple model to calculate $J_{\mathrm{C}}$ based on pinning properties of the voids (Fig. 4.26).

\subsubsection{Surface Reactivity}

QSE also plays an important role in the surface reactivity. Au is the most nobel element, still it becomes reactive at the nanometer scale [69]. In recent years, many experimental results have been published showing strong QSE modulations on ultrathin metal films [10, 11, 30, 70-73]. For example, Bartynski et al. reported thickness-dependent oscillations in the $\mathrm{CO}$ desorption from $\mathrm{Cu}$ films [11]. These oscillations were correlated with 2D subbands crossing the Fermi level periodically. Similarly, oxidation rate of ultrathin $\mathrm{Mg}(0001)$ exhibits oscillations as the film thickness is varied (Fig. 4.27) [10]. Maximum oxidation rate is achieved when a new quantum well state crosses the Fermi energy with increasing film thickness. These strong oscillations are related to the oscillatory decay lengths of the quantum well states, which affects the work function and electron transfer between the metal substrate and the anti-bonding orbitals of the incoming $\mathrm{O}_{2}$. Hence, the dissociation barrier and initial oxidation rate are ultimately controlled by QSE. Also, first-principles calculations were supplied to verify the validity of this basic mechanism [10].

Recently, STM observations of an oxidized $\mathrm{Pb}$ mesa in the quantum regime brought the most direct proof of the thickness modulated adsorption and reactivity 


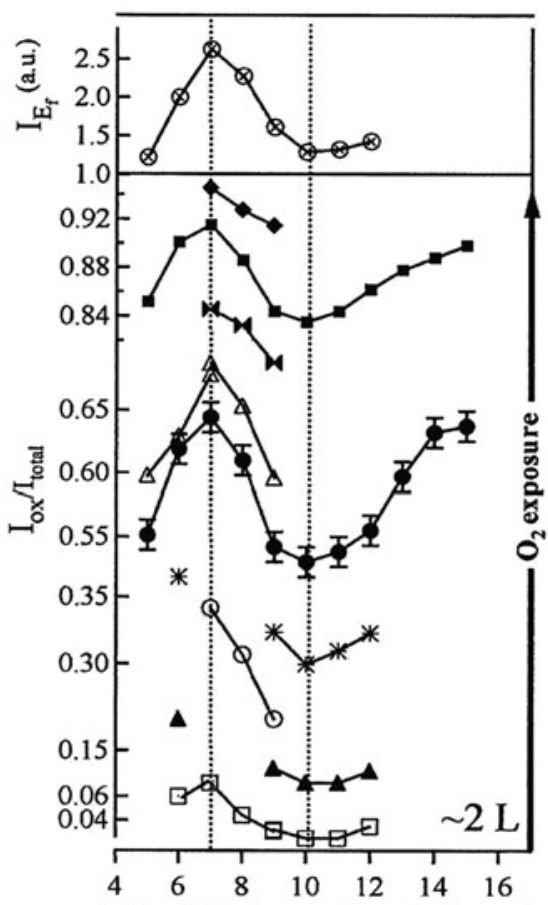

Film Thickness (atomic layers)

Fig. 4.27 Lower panel: plots of the Mg 2p oxide component in X-ray photoemission spectroscopy (XPS) as a function of film thickness obtained in several experimental runs. Data with the same symbol correspond to the same $\mathrm{O}_{2}$ exposure. Upper panel: photoemission intensity at the Fermi level. The observed tuning of the oxidation rate is directly correlated with the oscillations in the density of states at the Fermi level, due to the QSE. The oscillatory reactivity has been attributed to oscillatory dissociation barriers [10]

[30]. Fig. 4.28a shows the STM image of a flat-top Pb mesa grown on $\mathrm{Si}(111) 7 \times 7$ surface which accomodates $\mathrm{Pb}$ thicknesses between 10 and $16 \mathrm{ML}$ due to stepped substrate structure $\left(600 \times 600 \mathrm{~nm}^{2}\right.$ image $)$. Panel (b) shows the cros section of this mesa. Panel (d) shows the STM image of the thickness-dependent contrast developed after $60 \mathrm{~L}$ oxygen exposure at $100 \mathrm{~K}$ substrate temperature. From 9 to $12 \mathrm{ML}$ thick regions are labeled on the image. Bright spots are the chemisorbed oxygen clusters with a typical size of $1.2 \mathrm{~nm}$. Although the crystal orientation is the same $\mathrm{Pb}$ (111) for all the thicknesses, oxygen adsorption on even layers is clearly larger than that on the odd layers. This behavior is consistent with the aforementioned quantum electronic struture and local work function of the mesa [51]. Oxidation occurs at room temperature with additional oxygen exposure of $120 \mathrm{~L}$. This process again turns out to be thickness dependent and shown in panel (e). Panel (f) is a close up to the $\mathrm{PbO}$ nanocrystals.

So, both adsorption and oxidation are strongly enhanced when the $\mathrm{Pb}$ thickness is an even number of atomic layers. Quantitative analyses of the adsoption coverage 

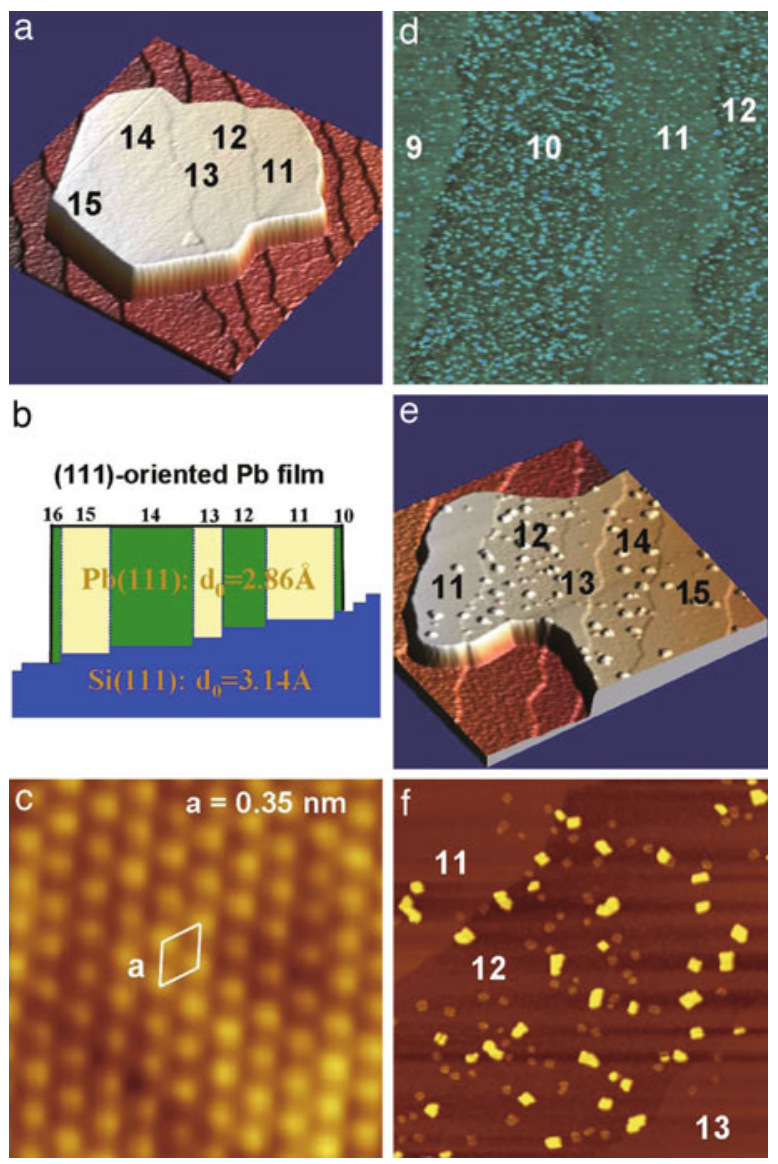

Fig. 4.28 (a) STM image of an atomically flat Pb mesa on a stepped Si surface. The numbers indicate the local thickness of the mesa in atomic layers. (b) Schematic cross section of the mesa. (c) Atomic resolution image on top of the mesa, revealing a $\mathrm{Pb}(111)$ orientation. (d-f) STM images during the various stages of adsorption and oxidation, as described in the text. Notice the contrasting behavior of the even and odd layers. Reproduced from [30]

indicate that the adsorption energy differs by about $9 \mathrm{meV}$ between 10 and $11 \mathrm{ML}$ films. In temperature scale this correspond to about $100 \mathrm{~K}$ difference between two adjacent thicknesses.

Local density of states (LDOS) and adsorption are shown to correlate in Fig. 4.29. The familiar crossover between even and odd layers takes place at around $17 \mathrm{ML}$ as expected from the same behavior of charge spill and work function. The correlation between the thickness-dependent LDOS and adsorption is explained in the framework of Newns-Anderson adsorption model [30, 74]: the chance of hybridization between the $\mathrm{s}-\mathrm{p}$ band of the $\mathrm{Pb}$ surface and the antibonding $\pi^{*}$ state of $\mathrm{O}_{2}$ is increased if the LDOS is higher. An STS study of quantum well states is also supplied by this reference. However, the correlation between the location of quantum 


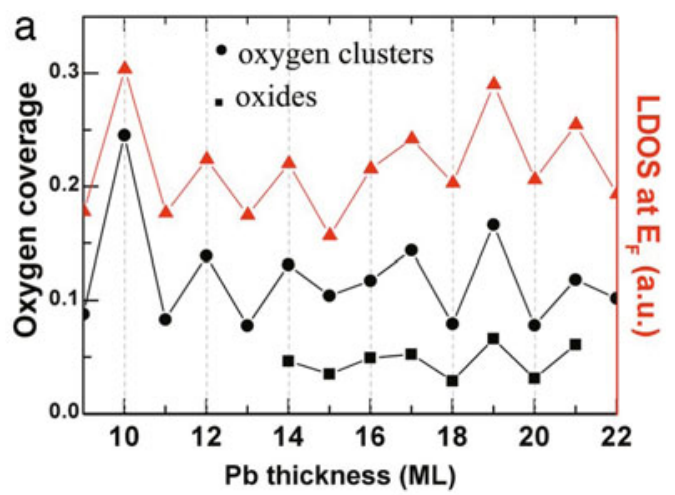

Fig. 4.29 Oxygen coverage in relation to local density of states at the Fermi level. Black dots show the oxygen clusters and black squares show the oxide coverage as defined in [30]. Red triangles show the local density of states. Reproduced from [30]

well states with respect to Fermi level and LDOS shown in Fig. 4.18 does not agree with the first-principles calculations given by [13].

\subsection{Tuning the Quantum Stability Via Alloying}

\subsubsection{Stability of PbBi Alloy Films}

In $\mathrm{Pb}(111)$ films, the periodic interruption of the bilayer growth follows a welldefined beating pattern with $9 \mathrm{ML}$, which is due to the fact that $\lambda_{\mathrm{F}}$ and $d$ are not exactly commensurate (see Sect. 4.3). This suggests the possibility of altering the quantum growth mode by tuning the Fermi wave vector $k_{\mathrm{F}}$ via doping [31]. According to the free electron model, the Fermi wave vector is given by $k_{\mathrm{F}}=\left(3 \pi^{2} n\right)^{1 / 3}$, where $n$ is the electron density. Bi has five valance electrons whereas $\mathrm{Pb}$ has four.

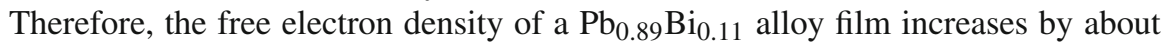
$3 \%$ compared to pure $\mathrm{Pb}$. This in turn increases the Fermi wave vector by $0.9 \%$ and, consequently, the associated beating period becomes $12.7 \mathrm{ML}$. The strong dependence of the beating wavelength on carrier density is due to the fact that $k_{\mathrm{F}}$ is very close to the middle of the second Brillion zone.

Figure 4.30 shows the STM images of $\mathrm{Pb}_{0.89} \mathrm{Bi}_{0.11}$ thin film alloys for various thicknesses. The alloy films exhibit a re-entrant bilayer-by-bilayer growth mode [31]. The observed stable thickness sequence is 4-6*7-9-11-13-15-17-19*20 . . ML, where ${ }^{*}$ indicates the even-odd crossover (this and all layer counts exclude a $1 \mathrm{ML}$ thick wetting layer; see [24]), the period of the even-odd crossovers is $\sim 13$ monolayers. Alloy films with higher $\mathrm{Bi}$ concentration $\left(\mathrm{Pb}_{0.80} \mathrm{Bi}_{0.20}\right)$ do not exhibit quantum growth anymore and simply follow classical layer-by-layer growth throughout the entire thickness range. This indicates that excessive scattering washes out the QSE. 


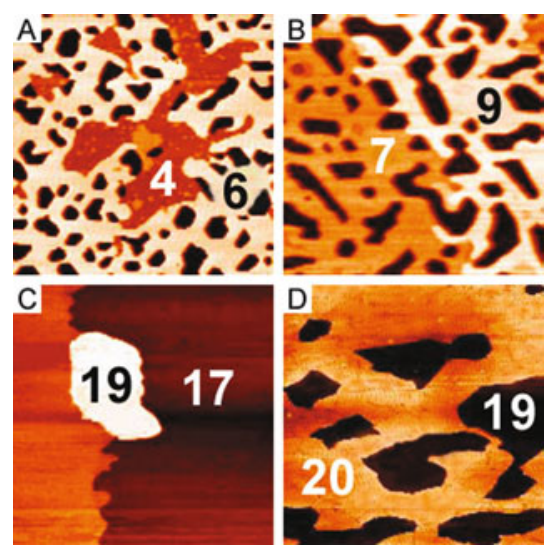

Fig. 4.30 STM images revealing the quantum growth mode of $\mathrm{Pb}_{0.89} \mathrm{Bi}_{0.11}$ alloy films, with bilayer growth (a) for 4 and $6 \mathrm{ML}$ thicknesses and (b) for 7 and $9 \mathrm{ML}$ thicknesses. In these images, the black pores are uncoated regions extending down to the substrate; in films used for study of superconductive properties, we verified the films to be simply connected and fully covering the substrate. Image (c) shows bilayer growth in a thicker film (also with a 1 ML terrace from the substrate), while (d) illustrates single layer growth near the even-odd crossover thickness. Reproduced from. [31]

DFT calculations for different $\mathrm{Bi}$ doping concentrations of $\mathrm{Pb}_{1-x} \mathrm{Bi}_{x}$ alloy films have been carried out $[31,75]$. Figure 4.31 shows the surface energy of $\mathrm{Pb}_{89} \mathrm{Bi}_{11}$ alloy films as a function of thickness for both freestanding films and films on a rotated $\mathrm{Si}(111)$ substrate. Note that for calculations performed on the substrate, both the beating periodicity and the location of the even-odd crossovers are in excellent agreement with the experimental result. Further calculations have been carried out

(A)

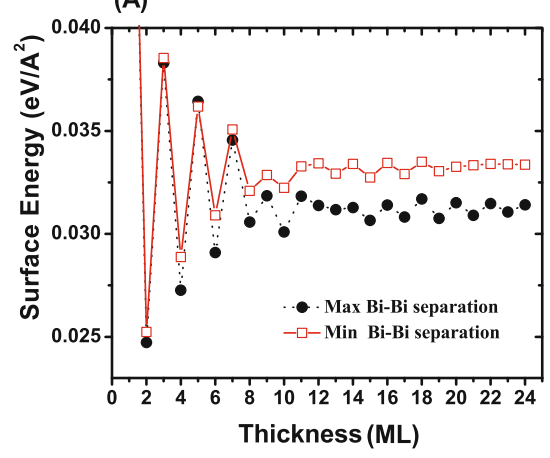

(B)

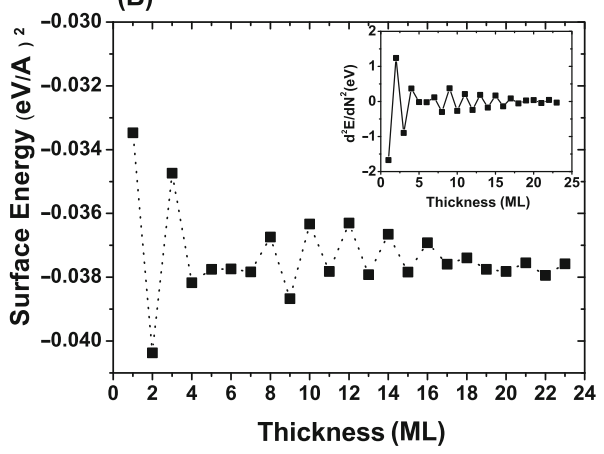

Fig. 4.31 (a) Surface energy of a $\mathrm{Pb}_{0.89} \mathrm{Bi}_{0.11}$ freestanding alloy slab obtained from first-principles DFT calculations. The black curve (circles) represents the surface energy for maximum Bi-Bi separation (within the constraints of a supercell calculation). The red curve (squares) represents the surface energy of a slab in which $\mathrm{Bi}$ atoms all have Bi nearest neighbors in the adjacent (111) layers; (b) surface energy of a $\mathrm{Pb}_{89} \mathrm{Bi}_{11}$ thin film alloy on a $\mathrm{Si}(111)$ substrate together with the second-order derivation of surface energy as a function of thickness (inset). Reproduced from [31] 
for different $\mathrm{Bi}$ concentrations [75]. $\mathrm{Pb}_{0.86} \mathrm{Bi}_{0.14}$ and $\mathrm{Pb}_{0.75} \mathrm{Bi}_{0.25}$ alloys yield 15 and $17 \mathrm{ML}$ beating periods, respectively. The QSE effect, however, gets weaker with increasing $\mathrm{Bi}$ content in agreement with the experimental results of $\mathrm{Pb}_{0.8} \mathrm{Bi}_{0.2}$ [31].

\subsubsection{Tuning the Superconductivity Via Alloying}

As reported earlier for clean $\mathrm{Pb}$ [14], the upper critical field $H_{\mathrm{c} 2}(T)$ markedly flattens near $T_{\mathrm{c}}$, resulting in a characteristic "hockey stick" profile that is particularly noticeable for the thinner films [31]. This feature becomes much more pronounced for the $\mathrm{Pb}_{89} \mathrm{Bi}_{11}$ and $\mathrm{Pb}_{80} \mathrm{Bi}_{20}$ quantum alloys compared to pure $\mathrm{Pb}$. The strong curvature near $T_{\mathrm{c}}$ marks a profound departure from the anisotropic Ginzburg-Landau theory, which predicts that $H_{\mathrm{c} 2} \propto\left(1-T / T_{\mathrm{c}}\right)$. The upper critical field resumes Ginzburg-Landau-like behavior below a characteristic temperature $T_{\mathrm{c}}^{*}$, which is defined by extrapolating the linear part of the low temperature $H_{\mathrm{c} 2}$ curve to zero dc field [31](see Fig. 4.32).

Although a rounding of $H_{\mathrm{c} 2}(T)$ near $T_{\mathrm{c}}$ is often attributed to structural inhomogeneities in conjunction with the boundary conditions for the pair wave function, the
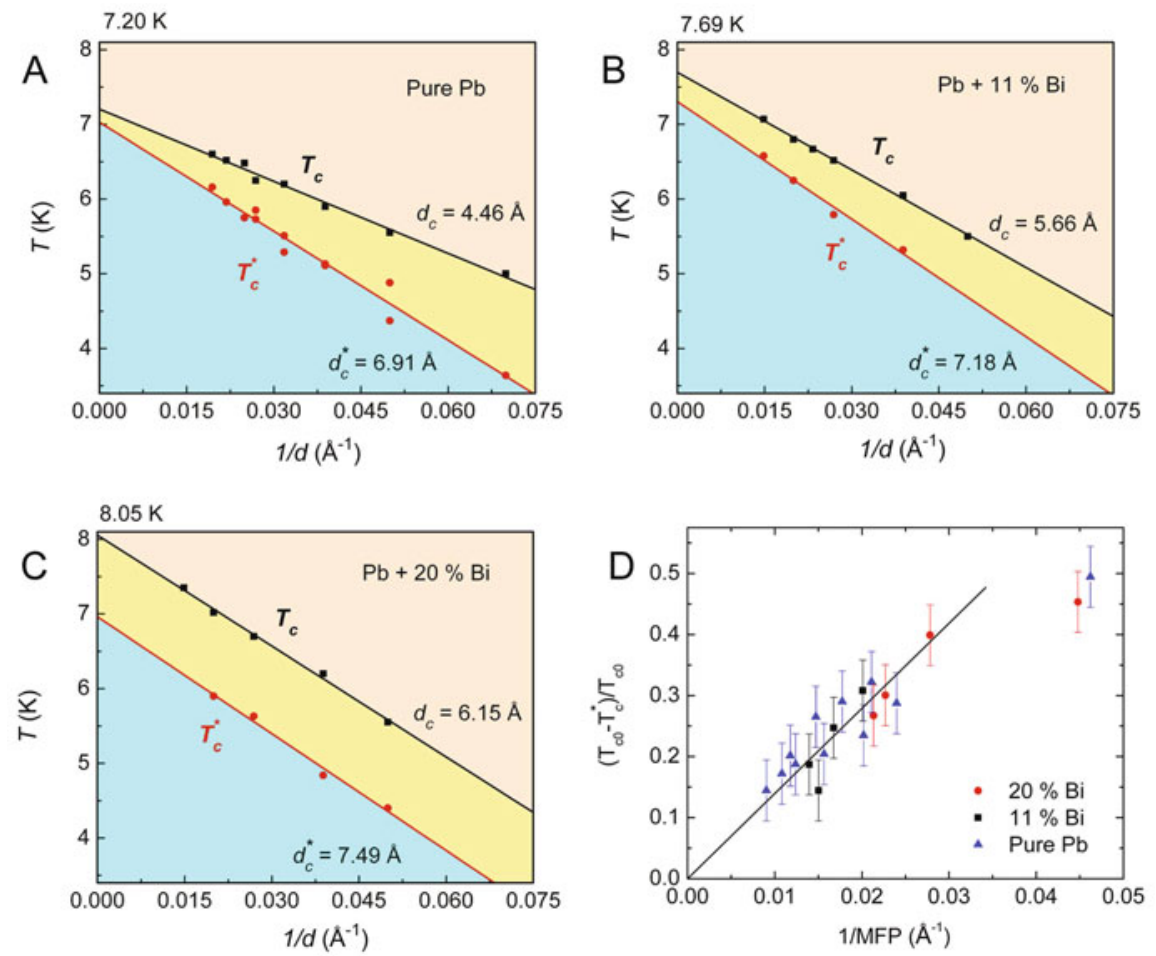

Fig. 4.32 Characteristic temperatures $T_{\mathrm{C}}$ and $T_{\mathrm{C}}^{*}$ values versus inverse film thickness (a) for pure $\mathrm{Pb}$, (b) for $\mathrm{Pb}_{0.89} \mathrm{Bi}_{0.11}$, and (c) for $\mathrm{Pb}_{0.80} \mathrm{Bi}_{0.20}$ alloys. Reproduced from [31] 
rounding here changes systematically with the fundamental nanoscale dimension $d$, because both $T_{\mathrm{c}}$ and $T_{\mathrm{c}}^{*}$ vary linearly with $1 / d$ while the low temperature slopes $\mathrm{d} H_{\mathrm{c} 2} / \mathrm{d} T \propto\left(d-d_{\mathrm{c}}^{*}\right)^{-1}$ also vary systematically with thickness [42]. On the other hand, the separation between $T_{\mathrm{c}}$ and $T_{\mathrm{c}}^{*}$ increases with Bi content, and $T_{\mathrm{c}}^{*}$ does not extrapolate to the $T_{\mathrm{c}}$ of the corresponding bulk alloys. This indicates that the thickness of the quantum alloy films is not the only parameter controlling $T_{\mathrm{c}}^{*}$.

It is interesting that the "hockey stick" profile of the upper critical field and breakdown of the anisotropic Ginzburg-Landau theory could signal multi-gap superconductivity related to the existence of multiple 2D subbands in the quantum alloys, where each band has its own intrinsic coherence length. This interpretation is based on an intriguing analogy with $\mathrm{MgB}_{2}$, where the slope of $H_{\mathrm{c} 2}(T)$ near $T_{\mathrm{C}}$ is determined primarily by intraband scattering in the "clean" $\sigma$ band and where a similar upturn in the upper critical field below $T_{\mathrm{C}}$ has been attributed to intraband scattering in the "dirty" band channel [76, 77]. In the PbBi alloy film, the $H_{\mathrm{c} 2}$ upturn would reflect a similar crossover phenomenon, possibly involving several orthogonal subband channels that are coupled via off-diagonal electron-phonon coupling and weak interband scattering [76, 77]. Because the intraband scattering relaxation time decreases with increasing subband index, the clean channel might be associated with the lowest subband. This conjecture could in principle be verified with low-temperature, ultrahigh-resolution scanning tunneling spectroscopy. In contrast to bulk $\mathrm{MgB}_{2}$ - one of the very few known multi-gap superconductors - such a low-dimensional superconductor would be fully tunable, which opens up new possibilities for experimental and theoretical studies of multi-gap superconductivity in the quantum regime.

\subsection{Concluding Remarks and Perspectives}

In this review, we attempted to highlight a novel type of "quantum engineering" in which quantum mechanics plays an essential role in defining the kinetic and thermodynamic parameters of metallic thin films as well as the preferred mode of growth of a variety of metallic nanostructures on semiconductor substrates. The impact of this new concept clearly lies in its enabling role. It provides the basis on which quantum size effects can be exploited to precisely control the formation of metallic structures; such structures formed in the quantum regime, in turn, are bound to serve as appealing platforms for elucidating intriguing quantum properties.

In the past 10 years, this field has evolved from the confirmation of the quantum stability concept in a wide variety of materials' systems to the discovery of their novel properties, which include magnetism [78], transport, superconductivity, work function, and surface chemical reactivity. Despite these significant advances, many challenging issues remain. For example, it is still not fully understand how the QWS formed in the normal direction affect the in-plane atomic motion in the film, particularly at the electronic and atomistic levels and the coupling between the two length scales. Further theoretical and experimental studies are needed for fully 
understanding the observed phenomena for precise structural control and exploring and designing noval physical properties in the quantum regime.

Further research efforts may also prove fruitful in the directions of optical adsorption, nano friction, catalysis, and reflectivity, all of which with clear relevance for sustainable energy applications. For example, it is expected that the QSE would have an strong modulation to the friction coefficient on $\mathrm{Pb}(111)$ and other ultrthin films, similar to the experimental observations showing that the electron friction can play a key role in the systems of $\mathrm{N}_{2}$ on the $\mathrm{Pb}$ substrate $[79,80]$.

It is also expected that future research in this area will shift toward synthesis of more complex metallic systems such as the quantum stabilization of "nano alloys" that are immiscible in bulk form [81, 82], as well as applications in other areas of chemistry, physics, and engineering. Intriguing possibilities include pushing the robustness of the superconducting state to even smaller length scales so as to obtain unprecedented insights into the emergence of collective quantum phenomena in structures containing the fewest number of atom [3]. As another example, conceptual advances in plasmonics could involve the tailoring of resonant frequencies and Landau damping in metallic nanoparticles with precisely controlled morphology (Özer et al., unpublished). Finally, molecular adsorption, decomposition, and chemical reactions on quantum mechanically confined metals and nano-alloy catalysts may lead to catalysis via quantum design. The authors hope that this review will contribute to the necessary cross-fertilization between the various research disciplines so that the enabling concept of quantum growth may find its way from traditional surface science to the broader science and engineering domain.

Acknowledgments During the course of this line of research we have benefited from many of our collaborators and colleagues in the field. In particular, we thank C. K. Shih, Q. Niu, J. H. Cho, A. R. Smith, J. R. Thompson, B. Wu, and E. J. Moon for their valuable contributions to the main findings and advances presented in this review. YJ acknowledges financial support from NSF of China (Grant No. 10574113, 10974182), HHW and ZYZ acknowledge financial support from NSF under contract No. DMR 06-06485 and DMR 0906025 and the US-DOE Office of Basic Energy Sciences, Division of Materials Sciences and Engineering.

\section{References}

1. D.C. Tsui, H.L. Stormer, A.C. Gossard, Two-dimensional magnetotransport in the extreme quantum limit. Phys. Rev. Lett. 48, 1559 (1982)

2. M.N. Baibich, J.M. Broto, A. Fert, F. Nguyen Van Dau, F. Petroff, P. Etienne, G. Creuzet, A. Friederich, J. Chazelas, Giant magnetoresistance of $(001) \mathrm{Fe} /(001) \mathrm{Cr}$ magnetic superlattices. Phys. Rev. Lett. 61, 2472 (1988)

3. A.R. Smith, K.-J. Chao, Q. Niu, C.-K. Shih, Formation of atomically flat silver films on GaAs with a "silver mean" quasi periodicity. Science 273, 226 (1996)

4. Z.Y. Zhang, Q. Niu, C.-K. Shih, "Electronic growth" of metallic overlayers on semiconductor substrates. Phys. Rev. Lett. 80, 5381 (1998)

5. V. Yeh, L. Berbil-Bautista, C.Z. Wang, K.M. Ho, M.C. Tringides, Role of the metal/semiconductor interface in quantum size effects: $\mathrm{Pb} / \mathrm{Si}(111)$. Phys. Rev. Lett. 85, 5158 (2000) 
6. C.M. Wei, M.Y. Chou, Theory of quantum size effects in thin $\mathrm{Pb}(111)$ films. Phys. Rev. B 66, $233408(2002)$

7. M.H. Upton, C.M. Wei, M.Y. Chou, T. Miller, T.C. Chiang, Thermal stability and electronic structure of atomically uniform Pb films on Si(111). Phys. Rev. Lett. 93, 026802 (2004)

8. M.M. Özer, Y. Jia, B. Wu, Z.Y. Zhang, H.H. Weitering, Quantum stability and reentrant bilayer-by-bilayer growth of atomically smooth $\mathrm{Pb}$ films on semiconductor substrates. Phys. Rev. B 72, 113409 (2005)

9. Y. Jia, B. Wu, H. H. Weitering, Z.Y. Zhang, Quantum size effects in $\mathrm{Pb}$ films from first principles: the role of the substrate. Phys. Rev. B 74, 035433 (2006)

10. L. Aballe, A. Barinov, A. Locatelli, S. Heun, M. Kiskinova, Tuning surface reactivity via electron quantum confinement. Phys. Rev. Lett. 93, 196103 (2004)

11. A.G. Danese, F.G. Curti, R.A. Bartynski, Quantum size effect induced modification of the chemisorption properties of thin metal films. Phys. Rev. B 70, 165420 (2004)

12. Y. Guo, Y.F. Zhang, X.Y. Bao, T.Z. Han, Z. Tang, L.X. Zhang, W.G. Zhu, E.G. Wang, Q. Niu, Z.Q. Qiu, J.F. Jia, Z.X. Zhao, Q.K. Xue, Superconductivity modulated by quantum size effects. Science 306, 1915 (2004)

13. D. Eom, S. Qin, M.Y. Chou, C.K. Shih, Persistent superconductivity in ultrathin Pb films: a scanning tunneling spectroscopy study. Phys. Rev. Lett. 96, 027005 (2006)

14. M.M. Özer, J.R. Thompson, H.H. Weitering, Hard superconductivity of a soft metal in the quantum regime. Nat. Phys. 2, 173 (2006)

15. M.M. Özer, J.R. Thompson, H.H. Weitering, Robust superconductivity in quantum-confined $\mathrm{Pb}$ : equilibrium and irreversible superconductive properties. Phys. Rev. B 74, 235427 (2006)

16. Z.G. Suo, Z.Y. Zhang, Epitaxial films stabilized by long-range forces. Phys. Rev. B 58, 5116 (1998)

17. R.C. Jakleic, J. Lambe, M. Mikkor, W.C. Vassell, Observation of electron standing waves in a crystalline box. Phys. Rev. Lett. 26, 88 (1971)

18. F.K. Schulte, A theory of thin metal films: electron density, potentials and work function. Surf. Sci. 55, 427 (1976)

19. P.J. Feibelman, Static quantum-size effects in thin crystalline, simple-metal films. Phys. Rev. B 27, 1991 (1983)

20. A. Zangwill, Physics at Surfaces (Cambridge University Press, Cambridge, 1998)

21. M. Hupalo, V. Yeh, L. Berbil-Bautista, S. Kremmer, E. Abram, M.C. Tringides, Uniformheight island growth of $\mathrm{Pb}$ on $\mathrm{Si}(111)-\mathrm{Pb}(\sqrt{ } 3 \times \sqrt{ } 3)$ at low temperatures. Phys. Rev. B 64, 155307 (2001)

22. H.W. Hong, C.M. Wei, M.Y. Chou, Z. Wu, L. Basile, H. Chen, M. Holt, T.C. Chiang, Alternating layer and island growth of $\mathrm{Pb}$ on $\mathrm{Si}$ by spontaneous quantum phase separation. Phys. Rev. Lett. 90, 076104 (2003)

23. van N. Stranski, L. Von Krastanow, Abhandlungen der Mathematisch-Naturwissenschaftlichen Klasse. Akademie der Wissenschaften und der Literatur in Mainz, 146, 797 (1939)

24. W.B. Su, S.H. Chang, W.B. Jian, C.S. Chang, L.J. Chen, T.T. Tsong, Correlation between quantized electronic states and oscillatory thickness relaxations of 2D Pb islands on $\mathrm{Si}(111)$ (7×7) surfaces. Phys. Rev. Lett. 86, 5116 (2001)

25. M. Hupalo, S. Kremmer, V. Yeh, L. Berbil-Bautista, E. Abram, M.C. Tringides, Uniform island height selection in the low temperature growth of $\mathrm{Pb} / \mathrm{Si}(111)-(7 \times 7)$. Surf. Sci. 493, 526 (2001)

26. M.M. Özer, C.-Z. Wang, Z. Zhang, H.H. Weitering, Quantum size effects in the growth, coarsening, and properties of ultra-thin metal films and related nanostructures. J. Low Temp. Phys. 157, 221 (2009)

27. B. Wu, Z. Zhang, Stability of metallic thin films studied with a free electron model. Phys. Rev. B 77, 035410 (2008)

28. M.C. Tringides, M. Jalochowski, E. Bauer, Quantum size effects in metallic nanostructures. Phys. Today 60, 50 (2007)

29. P. Czoschke, H. Hong, L. Basile, T.-C. Chiang, Quantum size effects in the surface energy of $\mathrm{Pb} / \mathrm{Si}(111)$ film nanostructures studied by surface $\mathrm{x}$-ray diffraction and model calculations. Phys. Rev. B 72, 075402 (2005) 
30. X. Ma, P. Jiang, Y. Qi, J. Jia, Y. Yang, W. Duan, W.-X. Li, X. Bao, S.B. Zhang, Q.-K. Xue, Experimental observation of quantum oscillation of surface chemical reactivities. Proc. Natl. Acad. Sci.104, 9204 (2007)

31. M.M. Özer, Y. Jia, Z.Y. Zhang, J.R. Thompson, H.H. Weitering, Tuning the quantum stability and superconductivity of ultrathin metal alloys. Science 316, 1594 (2007)

32. T.C. Chiang, Photoemission studies of quantum well states in thin films. Surf. Sci. Rep. 39, $181(2000)$

33. B.J. Hinch, C. Koziol, J.P. Toennies, G. Zhang, Evidence for quantum size effects observed by helium atom scattering during the growth of $\mathrm{Pb}$ on $\mathrm{Cu}(111)$. Europhys. Lett. 10, 341 (1989)

34. J. Braun, J.P. Toennies, Observation of a quantum size effect in the surface electron density of thin lead films. Surf. Sci. 384, L858, (1997)

35. R. Otero, A. L. Vázquez de Parga, R. Miranda, Observation of preferred heights in $\mathrm{Pb}$ nanoislands: a quantum size effect. Phys. Rev. B. 66, 115401 (2002)

36. J.R. Anderson, A.V. Gold, Fermi surface, pseudopotential coefficients, and spin-orbit coupling in lead. Phys. Rev. 139, A1459 (1965)

37. M. Jalochowski, H. Knoppe, G. Lilienkamp, E. Bauer, Photoemission from ultrathin metallic films: quantum size effect, electron scattering, and film structure. Phys. Rev. B 46, 4693 (1992)

38. J.C. Heyraud, J.J. Méois, Equilibrium shape and temperature; lead on graphite. Surf. Sci. 128, 334 (1983)

39. P. Czoschke, H.W. Hong, L. Basile, T.C. Chiang, Quantum beating patterns observed in the energetics of $\mathrm{Pb}$ film nanostructures. Phys. Rev. Lett. 93, 036103 (2004)

40. Y. Jia, B. Wu, C. Li, T. Einstein, H.H. Weitering, Z.Y. Zhang, Strong quantum size effects in $\mathrm{Pb}(111)$ thin films mediated by anomalous friedel oscillations. Phys. Rev. Lett. 105, 066101 (2010)

41. I.B. Altfeder, V. Narayanamurti, D.M. Chen, Imaging subsurface reflection phase with quantized electrons. Phys. Rev. Lett. 88, 206801 (2002)

42. P. Czoschke, H.W. Hong, L. Basile, T.C. Chiang, Quantum oscillations in the layer structure of thin metal films. Phys. Rev. Lett. 91, 226801 (2003)

43. J.H Cho, Ismail, Z.Y. Zhang, E.W. Plummer, Oscillatory lattice relaxation at metal surfaces. Phys. Rev. B 59, 1677 (1999)

44. M.W. Finnis, V. Heine, Theory of lattice contraction at aluminium surfaces. J. Phys. F. Met. Phys. 4, L37-L41 (1974)

45. A. Crottini, D. Cvetko, L. Floreano, R. Gotter, A. Morgante, F. Tommasini, Step height oscillations during layer-by-layer growth of $\mathrm{Pb}$ on $\mathrm{Ge}(001)$. Phys. Rev. Lett. 79, 1527 (1997)

46. L. Floreano, D. Cvetko, F. Bruno, G. Bavdek, A. Cossaro, R. Gotter, A. Verdini, A. Morgante, Quantum size effects in the low temperature layer-by-layer growth of $\mathrm{Pb}$ on $\mathrm{Ge}(001)$. Prog. Surf. Sci. 72, 135 (2003)

47. A. Mans, J.H. Dil, A. R. H. F. Ettema, H.H. Weitering, Quantum electronic stability and spectroscopy of ultrathin Pb films on Si(111)7×7. Phys. Rev. B 66, 195410 (2002)

48. M. Breitholtz, T. Kihlgren, S.-A. Lindgren, L. Wallden, Condensation of Na metal on graphite studied by photoemission. Phys. Rev. B 67, 235416 (2003)

49. C.J. Fall, N. Binggeli, A. Baldereschi, Work functions at facet edges. Phys. Rev. Lett. 88, $156802(2002)$

50. K. Besocke, B. Krahl-Urban, H. Wangner, Dipole moments associated with edge atoms; A comparative study on stepped Pt, Au and W surfaces. Surf. Sci. 68, 39-46 (1977)

51. Y. Qi, X. Ma, P. Jiang, S.H. Ji, Y.S. Fu, J.F. Jia, Q.K. Xue, S.B. Zhang, Atomic-layer-resolved local work functions of $\mathrm{Pb}$ thin films and their dependence on quantum well states. Appl. Phys. Lett. 90, 013109 (2007)

52. C.-S. Jiang, S.C. Li, H.B. Yu, D. Eom, X.D. Wang, Ph. Ebert, J.F. Jia, Q.K. Xue, C.K. Shih, Building Pb nanomesas with atomic-layer precision. Phys. Rev. Lett. 92, 106104 (2004)

53. T. Miller, M.Y. Chou, T.C. Chiang, Phase relations associated with one-dimensional shell effects in thin metal films. Phys. Rev. Lett. 102, 236803 (2009) 
54. F.J. Himpsel, J.E. Ortega, G.J. Mankey, R.F. Willis, Magnetic nanostructures. Adv. Phys. 47, $511(1998)$

55. M. Milum, P. Pervan, D.P. Woodruff, Quantum well structures in thin metal films: simple model physics in reality. Rep. Prog. Phys. 65, 99 (2002)

56. B. Allen, R.-C. Dynes, Transition temperature of strong-coupled superconductors reanalyzed. Phys. Rev. B 12, 905 (1975)

57. A. Crottini, D. Cvetko, L. Floreano, R. Gotter, A. Morgante, F. Tommasini, Step height oscillations during layer-by-layer growth of $\mathrm{Pb}$ on $\mathrm{Ge}(001)$. Phys. Rev. Lett. 79, 1527 (1997)

58. Y.F. Zhang, J.F. Jia, T.Z. Han, Z. Tang, Q.T. Shen, Y. Guo, Z.Q. Qiu, Q.K. Xue, Band structure and oscillatory electron-phonon coupling of $\mathrm{Pb}$ thin films determined by atomic-layer-resolved quantum-well states. Phys. Rev. Lett. 95, 096802 (2005)

59. F. Yndurain, M.P. Jigato, First principles calculation of localized surface phonons and electron-phonon interaction at $\mathrm{Pb}(111)$ thin films. Phys. Rev. Lett. 100, 205501 (2008)

60. J. Kosterlitz, D. Thouless, Ordering, metastability and phase transitions in two-dimensional systems. J. Phys. C 6, 1181 (1973)

61. D.B. Haviland, Y. Liu, A.M. Goldman, Onset of superconductivity in the two-dimensional limit. Phys. Rev. Lett. 62, 2180 (1989)

62. I. Vilfan, M. Henzler, O. Pfennigstorf, H. Pfnür, Anomalous thickness dependence of the hall effect in ultrathin Pb layers on Si(111). Phys. Rev. B. 66, 241306 (2002)

63. I. Vilfan, H. Pfnür, Charge-carrier transport properties of ultrathin Pb films. Eur. Phys. J. B. 36, $281(2003)$

64. J. Simonin, Surface term in the superconductive Ginzburg-Landau free energy: application to thin films. Phys. Rev. B 33, 7830 (1986)

65. R.C. Dynes, J.M. Rowell, Influence of electrons-per-atom ratio and phonon frequencies on the superconducting transition temperature of lead alloys. Phys. Rev. B 11, 1884 (1975)

66. S. Qin, J. Kim, Q. Niu, C.K. Shih, Superconductivity at the two-dimensional limit. Science 324, 1314 (2009)

67. H.H. Weitering, The world's thinnest superconductor. Chem. Phys.Chem. 10, 1 (2009)

68. G.J. Dolan, J. Silcox, Critical thicknesses in superconducting thin films. Phys. Rev. Lett. 30, 603 (1973)

69. M. Valden, X. Lai, D.W. Goodman, Onset of catalytic activity of gold clusters on titania with the appearance of nonmetallic properties. Science 281, 1647 (1998)

70. M.S. Chen, D. Kumar, C.-W. Yi, D.W. Goodman, The promotional effect of gold in catalysis by palladium-gold. Science 310, 291 (2005)

71. M.S. Chen, Y. Cai, Z. Yan, D.W. Goodman, On the origin of the unique properties of supported Au nanoparticles. J. Am. Chem. Soc. 128, 6341 (2006)

72. M.S. Chen, D.W. Goodman, Structure-activity relationships in supported Au catalysts. Catal. Today 111, 22-23 (2006)

73. N. Binggeli, M. Altarelli, Surface reactivity and quantum-size effects on the electronic density decay length of ultrathin metal films. Phys. Rev. Lett. 96, 036805 (2006)

74. J.K. Norskov, Chemisorption on metal surfaces. Rep. Prog. Phys. 53, 1253 (1990)

75. N. Trivedi, N.W. Ashcroft, First-principles study of quantum size effects in ultrathin Pb-Bi metal alloy films. Phys. Rev. B. 38, 12298 (1988)

76. A. Gurevich, S. Patnaik, V. Braccini, K.H. Kim, C. Mielke, X. Song, L.D. Cooley, S.D. Bu, D.M. Kim, J.H. Choi, L.J. Belenky, J. Giencke, M.K. Lee, W. Tian, X.Q. Pan, A. Siri, E.E. Hellstrom, C.B. Eom, D.C. Larbalestier, Very high upper critical fields in $\mathrm{MgB}_{2}$ produced by selective tuning of impurity scattering. Supercond. Sci. Tech. 17, 278 (2004)

77. M. Mansor, J.P. Carbotte, Upper critical field in two-band superconductivity. Phys. Rev. B 72, $024538(2005)$

78. Z.Q. Qiu, N.V. Smith, Quantum well states and oscillatory magnetic interlayer coupling. J. Phys Condens. Matter 14, R169 (2002)

79. A. Dayo, W. Alnasrallah, J. Krim, Superconductivity-dependent sliding friction. Phys. Rev. Lett. 80, 1690 (1998) 
80. M. Highland, J. Krim, Superconductivity dependent friction of water, nitrogen, and superheated He films adsorbed on Pb(111). Phys. Rev. Lett. 96, 226107 (2006)

81. E.J. Moon, M. M. Özer, R.J. Thompson, H.H. Weitering, submitted

82. A. Khajetoorians, W.G. Zhu, J.S. Kim, S. Qin, H. Eisele, Z.Y. Zhang, C.-K. Shih, Adsorbateinduced restructuring of $\mathrm{Pb}$ mesas grown on vicinal $\mathrm{Si}(111)$ in the quantum regime. Phys. Rev. B 80, 245426 (2009) 